Submitted to: J. Phys. A: Math. Gen.

\title{
Discrete breathers in a two-dimensional Fermi-Pasta-Ulam lattice
}

\author{
Imran A Butt and Jonathan A D Wattis \\ Theoretical Mechanics, School of Mathematical Sciences, University of Nottingham, \\ University Park, Nottingham, NG7 2RD, UK \\ E-mail: imran.butt@maths.nott.ac.uk, jonathan.wattis@nottingham.ac.uk
}

\begin{abstract}
Using asymptotic methods, we investigate whether discrete breathers are supported by a two-dimensional Fermi-Pasta-Ulam lattice. A scalar (one-component) two-dimensional Fermi-Pasta-Ulam lattice is shown to model the charge stored within an electrical transmission lattice. A third-order multiple-scale analysis in the semidiscrete limit fails, since at this order, the lattice equations reduce to the $(2+1)$ dimensional cubic nonlinear Schrödinger (NLS) equation which does not support stable soliton solutions for the breather envelope. We therefore extend the analysis to higher order and find a generalised $(2+1)$-dimensional NLS equation which incorporates higher order dispersive and nonlinear terms as perturbations. We find an ellipticity criterion for the wave numbers of the carrier wave. Numerical simulations suggest that both stationary and moving breathers are supported by the system. Calculations of the energy show the expected threshold behaviour whereby the energy of breathers does not go to zero with the amplitude; we find that the energy threshold is maximised by stationary breathers, and becomes arbitrarily small as the boundary of the domain of ellipticity is approached.
\end{abstract}

PACS numbers: 05.45.-a, 05.45.Yv

\section{Introduction}

Discrete breathers (DBs, or simply, breathers) are time-periodic and spatially localised exact solutions of the equations of motion for a network of coupled nonlinear oscillators. In this paper we investigate the form, properties and behaviour of breathers in two dimensional systems with square symmetry by using asymptotic expansions to determine the governing equation of an envelope which modulates a plane wave. A perturbed nonlinear Schrödinger equation is obtained. The resulting approximations to breathers are used as initial conditions in numerical simulations of the Fermi-Pasta-Ulam (FPU) lattice [1], and the breather modes are observed to persist with very little change of shape over a considerable period of time. A detailed description of structure of paper is given at the end of this section; the remainder of this section is given over to a review of the relevant literature on discrete breathers. 
The discreteness of the lattice paired with its nonlinearity allows for the existence of DB solutions in a lattice. Specifically, in order to be long-lived, a breather's frequency (and that of its harmonics) must avoid resonances with the phonon band. The discreteness of the system gives rise to gaps and cut-offs in the phonon spectrum, while the nonlinearity allows for the shifting of the frequency of the breather (and its harmonics) outside of the phonon band, thus avoiding resonances. There is a large body of work on DBs (sometimes referred to as anharmonic localised modes, localised oscillations, nonlinear localised excitations etc.). Much of the early work is heuristic, being based on approximate analytic methods such as the Rotating Wave Approximation [2], or numerical procedures, for instance [3]. Ovchinnikov [4] gives an early discussion of DBs; for a comprehensive review of the subject, see, for instance, Flach [5].

Early work on discrete breathers in higher dimensional systems includes that of Takeno [6], who introduces lattice Green's functions to find approximations to breather solutions in one-, two- and three-dimensional lattices. A similar method is applied in [7] to find the properties and profiles of localised modes in general $d$ dimensional lattices. Other authors present numerically-obtained breather solutions in two-dimensional lattices. For example, Burlakov et al. [8] find breather solutions in a two-dimensional square lattice with cubic and quartic nonlinearity, and Bonart et al. [9] show numerical simulations of localised excitations in one, two- and three-dimensional scalar lattices (that is, lattices with one degree of freedom at each site). The stability of these modes is also investigated. In addition, Tamga et al. [10] and Ovchinnikov \& Flach [11] also address higher dimensional systems using analytical approaches.

In 1994 Mackay and Aubry [12] extended the interest in DBs by rigorously establishing the existence of stationary breathers, not only in one-, but also in higherdimensional systems. They began by considering a specific model, namely a onedimensional Hamiltonian lattice with linear coupling between nearest neighbours, and a nonlinear on-site potential (the Klein-Gordon lattice is an example of such a model). The existence of DB solutions is established by the method of an anti-continuum limit, first introduced by Aubry and Abramovici [13]. The remarkable aspect of the proof is that it holds provided only rather weak hypotheses are met. Of direct relevance to the present work, it is shown that breathers can exist in lattices of any dimension, subject to only minimal additional constraints.

The origins and features of localised excitations in Klein-Gordon lattices are investigated by Flach \& Willis [14, 15]. Flach et al. [16] show that the existence and fundamental properties of localised excitations carry across from one-dimensional to twodimensional lattices. They argue that the theory described in [14, 15] holds regardless of lattice dimension. Hence the existence of DBs is predicted in three-dimensional lattices and higher. In fact, this conjecture is confirmed by Mackay and Aubry's work [12].

While some fundamental properties such as the existence of DBs are not affected by lattice dimension, other properties do depend strongly upon this, for instance, the energetic properties of DBs. We briefly describe results on $d$-dimensional hypercubic lattices of $N$ sites. For Hamiltonian systems, stationary breathers occur in one- 
parameter families. Suitable parameters include, for instance, the energy $E$, frequency $\omega_{b}$, or amplitude of the breather. In many systems, breathers of arbitrarily small amplitude can be found, although this is not necessarily so (see Aubry et al. [17], for example). In the limit of small amplitude, DBs typically widen and approach the solutions of the linearised lattice equations. However, the breather frequency must also be nonresonant with the phonon band, that is, $\omega_{q} / \omega_{b} \notin \mathbb{N}$. These two requirements can be fulfilled only if the breather frequency $\omega_{b}$ tends to an edge $\omega_{q}$ of the phonon spectrum as amplitude tends to zero. This observation suggests a link between discrete breathers and phonon modes, namely that breathers appear through a bifurcation from a phonon mode. So far, this has been proved only for specific one-dimensional lattices (see James [18]), and numerical work supports the conjecture in others (Sandusky \& Page [19]). Assuming this conjecture to be true, Flach [20] calculates the energy $E_{c}$ at which this bifurcation occurs. The quantity $E_{c}$ represents the minimum energy of DBs in the lattice. One might expect $E_{c}=0$ as occurs in one-dimensional lattices; however, in higher dimensions it is possible that $E_{c}>0$. Then no breathers with energies $E$ in the range $0<E<E_{c}$ exist. For an infinite lattice, as $N \rightarrow \infty$, different limiting values of $E_{c}$ are obtained depending on the dimension of the lattice: as described by Flach et al. [21], one obtains $E_{c} \sim N^{1-2 / d}$. Thus for $d<2$, breathers of arbitrarily small energy can be found. However, for $d \geq 2$, breather energies do not approach zero even as the amplitude tends to zero. In other words, there exists a positive lower bound on the energy of DBs. In calculating $E_{c}$, Flach [20] assumes the on-site and nearest-neighbour interaction potentials to be infinitely differentiable. Kastner [22] obtains estimates for the energy $E_{c}$ in the degenerate case where the interaction and on-site potentials are not smooth but only twice continuously differentiable (that is, $C^{2}$ but not $C^{3}$ ). In this unusual case, it can be shown that $E_{c} \sim N^{1-4 / d}$. Hence, in the limit $N \rightarrow \infty$, for $d<4$, such systems can support breathers of arbitrarily small energy. However, for $d \geq 4$, there exists a positive lower bound on the energy of DBs.

We mention an interesting series of papers by Marin, Eilbeck and Russell who investigate breather mobility in two-dimensional lattices with differing geometries. Their work is motivated by the observation of dark lines or "tracks" along crystal directions in white mica (see Russell [23]). An extensive account of the track forming process is given by Marin et al. [24], who also posit that breather modes are responsible for trackcreation. Marin et al. [25] numerically simulate the hexagonal Potassium planes within mica. Their results suggest that moving breathers do exist, and that the lattice exhibits a strong directional preference whereby breathers travel only along lattice directions. Breathers are easily generated in the lattice by imparting an initial velocity to a few consecutive atoms. After an initial transient (wherein a small amount of energy is radiated), a robust breather, slightly elongated in shape, emerges and travels with almost no further change in shape. The breather is stable against lateral spreading, with perhaps one or two atoms oscillating in the direction perpendicular to the line of travel. Along the breather path, typically no more than three or four consecutive atoms oscillate. The initial velocities can be directed as much as $\pm 15^{\circ}$ from a lattice direction, 
and a moving breather still emerges along the crystal axis. Larger deflections (around $\pm 30^{\circ}$ ) result in two breathers, each moving along the nearest lattice axes. However, it is not possible to generate breathers which travel in directions other than these. Similar results (termed "quasi-one-dimensional" effects, see Russell \& Collins [26]) are obtained by Marin et al. [27] in a later study on two- and three- dimensional lattices with different geometries.

While a good deal literature is available for breathers in higher-dimensional systems, much of the rigorous work is not concerned with determining profiles of breather solutions; such work has been extensive for one-dimensional systems (see Remoissenet [28], Kivshar \& Malomed [29]). The aim of this paper is thus to provide analytical results on the profiles of breathers in a higher dimensional system.

In the next section, we aim to find a leading order asymptotic form for a restricted class of breather solutions for the two-dimensional FPU equation, namely, those of small amplitude and with slowly varying envelope. We apply the method of multiple scales to (2.3), much the same as in our earlier work on breather solutions in onedimensional FPU lattices (Butt \& Wattis [30]). Unfortunately, while the multiple scales approach is extremely productive for the one-dimensional case, a similar analysis is made more complicated by the presence of two spatial dimensions. This is because, at third order, the two-dimensional FPU lattice equations reduce to a scalar cubic twodimensional nonlinear Schrödinger (NLS) equation. Unlike its one-dimensional analogue for which analytic formulae for soliton solutions are known (and give the form of the breather envelope), the cubic NLS equation over $\mathbb{R}^{2}$ does not support stable soliton solutions, only the unstable Townes soliton (Chiao et al. [31]). When subjected to small perturbations, these may blow up (the amplitude diverges in finite time), or disperse completely. However, it is known that unstable Townes solitons may be stabilised by including higher order effects in the model. In Section 3 we extend our asymptotic analysis to incorporate higher order dispersive and nonlinear terms. We show that the two-dimensional ETL equations reduce to a generalised nonlinear Schrödinger equation which includes terms known to stabilise the breather solution. We carry out extensive numerical simulations (presented in Section 4), demonstrating the stability and longlived nature of breathers in the two-dimensional FPU system. In Section 5, we review the progress made in this tentative multiple-scale approach to finding breathers in twodimensional systems. Some of the principal difficulties that have arisen are discussed, and we outline the ways in which these may be overcome.

\section{A two-dimensional Fermi-Pasta-Ulam lattice}

\subsection{Preliminaries}

In the present work, we investigate whether discrete breathers are supported by a scalar (one-component) two-dimensional electrical transmission lattice (ETL). This lattice comprises a square configuration of repeating units, each made up of two linear inductors 
and a nonlinear capacitor. It is straightforward to show that the evolution of charge stored by each capacitor is governed by a two-dimensional version of the Fermi-PastaUlam (FPU) equations, which we now show form a natural extension of the classic one-dimensional system studied by Fermi et al [1]. Breathers in one-dimensional FPU systems have been studied extensively; this system is described by

$$
H=\sum_{n} \frac{1}{2} s_{n}^{2}+V\left(r_{n+1}-r_{n}\right)
$$

which implies

$$
\frac{\mathrm{d}^{2} r_{n}}{\mathrm{~d} t^{2}}=V^{\prime}\left(r_{n+1}-r_{n}\right)-V^{\prime}\left(r_{n}-r_{n-1}\right)
$$

where $V(\cdot)$ is some interaction potential. The anti-continuum limit method of Mackay and Aubry [12] is inapplicable for such systems, since FPU lattices do not possess an uncoupled limit in which trivial breathers can be found. Nevertheless, early numerical and analytical work, much of it admittedly ad hoc, suggested that DBs are indeed supported by 1D FPU lattices (see for instance, Takeno et al. [2]). A rigorous existence proof for DB solutions is given by James [18].

A reformulation of (2.2) using $q_{n}:=r_{n+1}-r_{n}$ yields

$$
\frac{\mathrm{d}^{2} q_{n}}{\mathrm{~d} t^{2}}=V^{\prime}\left(q_{n+1}\right)-2 V^{\prime}\left(q_{n}\right)+V^{\prime}\left(q_{n-1}\right)
$$

From this form, a second Hamiltonian form distinct from (2.1) becomes apparent, namely

$$
\widetilde{H}=\sum_{n} \frac{1}{2}\left(p_{n+1}-p_{n}\right)^{2}+V\left(q_{n}\right)
$$

In this form, the nearest-neighbour coupling and nonlinearity have been separated; the coupling now appearing in a harmonic term and the nonlinearity involving only one site.

We now show that $\widetilde{H}(2.4)$ and $H(2.1)$ are numerically equal even though they appear to arise from different Hamiltonian formulations. We introduce forward and backward difference operators $\delta^{ \pm}$defined by $\delta^{+} \Phi_{n}=\Phi_{n+1}-\Phi_{n}$ and $\delta^{-} \Phi_{n}=\Phi_{n}-\Phi_{n-1}$. Then $\delta^{2}$, defined by $\delta^{2} \Phi_{n}=\Phi_{n+1}-2 \Phi_{n}+\Phi_{n-1}$, satisfies $\delta^{2}=\delta^{+} \delta^{-}=\delta^{-} \delta^{+}$. Hamilton's equations applied to (2.4) imply $\dot{q}_{n}=-\delta^{2} p_{n}$. Since $q_{n}:=\delta^{+} r_{n}$ we have $\delta^{+} \dot{r}_{n}=\delta^{+} \delta^{-}\left(-p_{n}\right)$, so $\dot{r}_{n}=s_{n}=C-\delta^{-} p_{n}$ for some quantity $C$, independent of $n$. Now consider $2(\widetilde{H}-H)=\sum_{n}\left(p_{n+1}-p_{n}\right)^{2}-\sum_{n} s_{n}^{2}$; then $2(\widetilde{H}-H)=-\sum_{n} C \quad$ (assuming that both $H$ and $\widetilde{H}$ are finite, and that $\left.\lim _{n \rightarrow \pm \infty} p_{n}=0\right)$. Hence, we deduce $C=0$, and so $H=\widetilde{H}$.

\subsection{Derivation of model equations}

The two-dimensional electrical transmission lattice (ETL) comprises a network of repeating unit sections, each consisting of two identical linear inductors and a nonlinear capacitor. The arrangement is illustrated in Figure 2.1. We define lattice nodes by the locations of capacitors. The area surrounding the $(m, n)$ th capacitor of the twodimensional ETL is illustrated in Figure 2.2. 


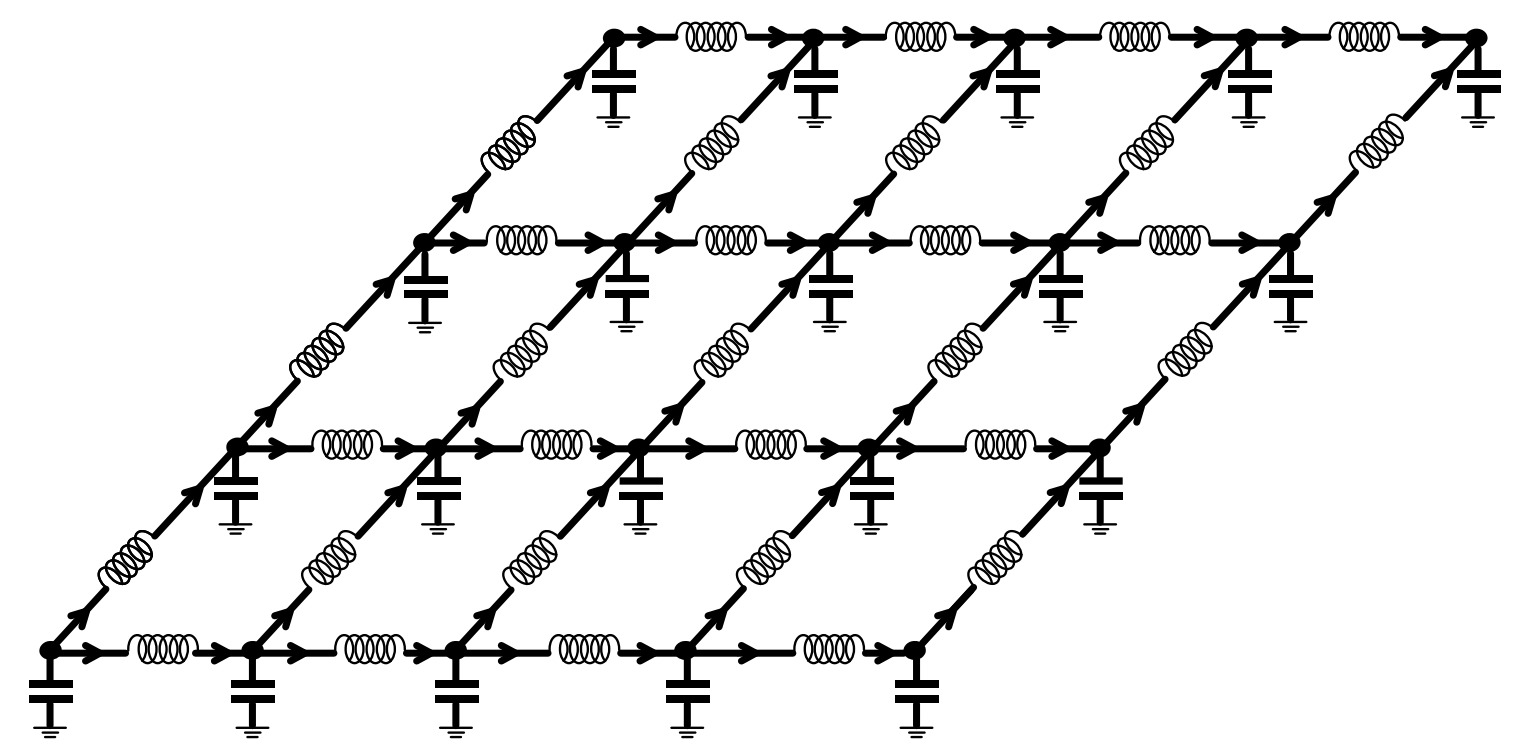

Figure 2.1. The two-dimensional electrical transmission lattice (2D ETL)

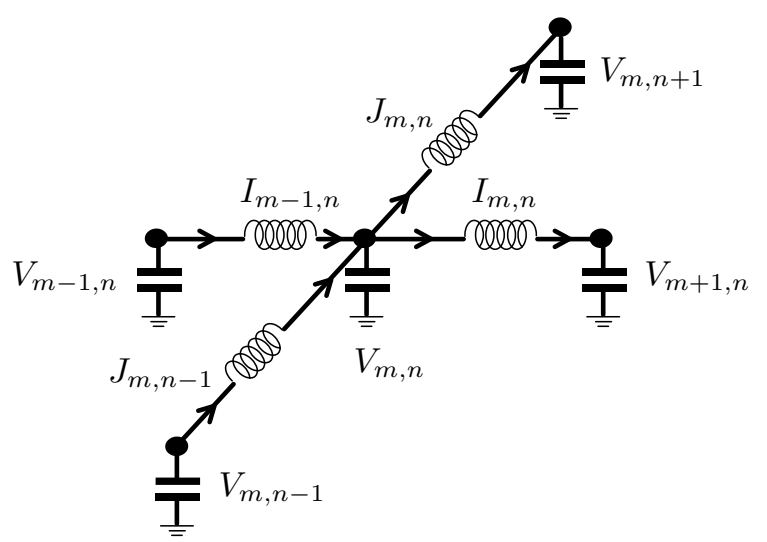

Figure 2.2. Enlarged view of the $2 \mathrm{D}$ ETL at the $(m, n)$ th node

The variable $V_{m, n}(t)$ denotes the voltage across the $(m, n)$ th capacitor, $Q_{m, n}(t)$ denotes the charge stored on the $(m, n)$ th capacitor, $I_{m, n}(t)$ denotes the current through the $(m, n)$ th inductor that lies horizontally in the plane of the lattice, and $J_{m, n}(t)$ denotes the current through the $(m, n)$ th inductor that lies in the plane of the lattice perpendicular to $I_{m, n}$. First, the equations governing voltage $V_{m, n}$, current $I_{m, n}$ and $J_{m, n}$, and charge $Q_{m, n}$ in the lattice are derived. Considering the section of the lattice shown in Figure 2.2 and applying Kirchoff's law, the difference in shunt voltage at the $(m, n)$ th and $(m+1, n)$ th sites is given by

$$
V_{m+1, n}-V_{m, n}=-L \frac{\mathrm{d} I_{m, n}}{\mathrm{~d} t}
$$

and the difference in shunt voltage at the $(m, n)$ th and $(m, n+1)$ th sites is given by

$$
V_{m, n+1}-V_{m, n}=-L \frac{\mathrm{d} J_{m, n}}{\mathrm{~d} t},
$$


where the inductance $L$ is a constant. Since the total charge is conserved, we also have that

$$
I_{m-1, n}+J_{m, n-1}-I_{m, n}-J_{m, n}=\frac{\mathrm{d} Q_{m, n}}{\mathrm{~d} t} .
$$

Using (2.5), (2.6) and (2.7), we find that

$$
\left(\delta_{m}^{2}+\delta_{n}^{2}\right) V_{m, n}=L \frac{\mathrm{d}^{2} Q_{m, n}}{\mathrm{~d} t^{2}}
$$

where $\delta_{m}^{2}$ and $\delta_{n}^{2}$ denote the second difference operators defined by $\delta_{m}^{2} A_{m, n}=A_{m+1, n}-$ $2 A_{m, n}+A_{m-1, n}$ and $\delta_{n}^{2} A_{m, n}=A_{m, n+1}-2 A_{m, n}+A_{m, n-1}$ respectively.

The nonlinear capacitance is a function of the voltage, and for small voltages, the capacitance-voltage relationship can be approximated by a polynomial expansion

$$
C\left(V_{m, n}\right)=C_{0}\left(1+2 \alpha V_{m, n}+3 \beta V_{m, n}^{2}+4 \gamma V_{m, n}^{3}+5 \delta V_{m, n}^{4}\right),
$$

where $C_{0}, \alpha, \beta, \gamma$ and $\delta$ are constants. Since the capacitance $C_{m, n}$ is defined by $C_{m, n}=\mathrm{d} Q_{m, n} / \mathrm{d} V_{m, n}$, it follows that

$$
Q_{m, n}=C_{0}\left(V_{m, n}+\alpha V_{m, n}^{2}+\beta V_{m, n}^{3}+\gamma V_{m, n}^{4}+\delta V_{m, n}^{5}\right) .
$$

Hence (2.8) becomes

$$
\left(\delta_{m}^{2}+\delta_{n}^{2}\right) V_{m, n}=L C_{0}\left(V_{m, n}+\alpha V_{m, n}^{2}+\beta V_{m, n}^{3}+\gamma V_{m, n}^{4}+\delta V_{m, n}^{5}\right)^{\prime \prime},
$$

where the prime denotes differentiation with respect to time. By choosing a suitable timescale, we may put $L C_{0}=1$ without loss of generality.

We choose to rewrite the equations (2.11) in terms of the variable $Q_{m, n}$ instead of $V_{m, n}$. The reason for this is that (2.11) takes on the more familiar form of the onedimensional FPU lattice equations (2.2). Firstly, $V_{m, n}$ must be found in terms of $Q_{m, n}$. Equation (2.10) is a quintic polynomial in $V_{m, n}$ which must be inverted to give $V_{m, n}$ in terms of $Q_{m, n}$. Since we are interested only in leading order solutions to (2.11), we may approximate the exact expression for $V_{m, n}$ by a quintic polynomial in $Q_{m, n}$. Hence we assume the following expansion for $V_{m, n}$

$$
V_{m, n}=V\left(Q_{m, n}\right) \sim \frac{Q_{m, n}}{C_{0}}+\frac{a^{\prime} Q_{m, n}^{2}}{C_{0}^{2}}+\frac{b^{\prime} Q_{m, n}^{3}}{C_{0}^{3}}+\frac{c^{\prime} Q_{m, n}^{4}}{C_{0}^{4}}+\frac{d^{\prime} Q_{m, n}^{5}}{C_{0}^{5}},
$$

where $a^{\prime}, b^{\prime}, c^{\prime}$ and $d^{\prime}$ are combinations of $\alpha, \beta, \gamma$ and $\delta$ obtained by substituting the expansion for $V_{m, n}$ (2.12) into (2.10) and matching coefficients of corresponding powers of $Q_{m, n}$. Proceeding thus, we find that

$$
\begin{aligned}
& a^{\prime}=-\alpha, \\
& b^{\prime}=-\beta+2 \alpha^{2}, \\
& c^{\prime}=-\gamma-5 \alpha\left(\alpha^{2}-\beta\right), \\
& d^{\prime}=-\delta+6 \alpha \gamma-21 \alpha^{2} \beta+3 \beta^{2}+14 \alpha^{4} .
\end{aligned}
$$

Finally, rewriting (2.11) in terms of $Q_{m, n}$ gives the equations governing charge $Q_{m, n}$ in the lattice

$$
\frac{\mathrm{d}^{2} Q_{m, n}}{\mathrm{~d} t^{2}}=\left(\delta_{m}^{2}+\delta_{n}^{2}\right)\left[Q_{m, n}+a Q_{m, n}^{2}+b Q_{m, n}^{3}+c Q_{m, n}^{4}+d Q_{m, n}^{5}\right]
$$


where $a=a^{\prime} / C_{0}, b=b^{\prime} / C_{0}^{2}, c=c^{\prime} / C_{0}^{3}$ and $d=d^{\prime} / C_{0}^{4}$. Equation (2.14) is a twodimensional analogue of the well known one-dimensional FPU equations (Fermi et al. [1]). It is worth noting that the lattice equations (2.14) can be derived from the following Hamiltonian

$$
\widetilde{H}=\sum_{m, n} \frac{1}{2}\left(P_{m+1, n}-P_{m, n}\right)^{2}+\frac{1}{2}\left(P_{m, n+1}-P_{m, n}\right)^{2}+\mathcal{F}\left(Q_{m, n}\right)
$$

where $\mathcal{F}\left(Q_{m, n}\right)$ satisfies $\mathcal{F}^{\prime}\left(Q_{m, n}\right)=V\left(Q_{m, n}\right)$ (see $\left.(2.12)\right)$, and $P_{m, n}, Q_{m, n}$ are canonically conjugate momenta and displacement of the system, with

$$
\frac{\mathrm{d} Q_{m, n}}{\mathrm{~d} t}=-\left(\delta_{m}^{2}+\delta_{n}^{2}\right) P_{m, n}, \quad \frac{\mathrm{d} P_{m, n}}{\mathrm{~d} t}=-\mathcal{F}^{\prime}\left(Q_{m, n}\right) .
$$

The Hamiltonian (2.15) is analogous to the Hamiltonian (2.4) of the one-component system; however, we have been unable to find a Hamiltonian of the two-dimensional system which is analogous to the one-dimensional form (2.1).

\subsection{Asymptotic analysis}

We seek breather solutions of the system of equations (2.14). Of course, no explicit analytic expression can be found for breather solutions, and so asymptotic methods are used to determine an approximate analytic form. We apply the method of multiple scales, and introduce new variables defined by

$$
X=\epsilon m, \quad Y=\epsilon n, \quad \tau=\epsilon t \quad \text { and } \quad T=\epsilon^{2} t .
$$

We look for solutions of the form

$$
\begin{aligned}
Q_{m, n}(t)= & \epsilon \mathrm{e}^{i \psi} F(X, Y, \tau, T)+\epsilon^{2} G_{0}(X, Y, \tau, T)+\epsilon^{2} \mathrm{e}^{i \psi} G_{1}(X, Y, \tau, T) \\
& +\epsilon^{2} \mathrm{e}^{2 i \psi} G_{2}(X, Y, \tau, T)+\epsilon^{3} H_{0}(X, Y, \tau, T)+\epsilon^{3} \mathrm{e}^{i \psi} H_{1}(X, Y, \tau, T) \\
& +\epsilon^{3} e^{2 i \psi} H_{2}(X, Y, \tau, T)+\epsilon^{3} \mathrm{e}^{3 i \psi} H_{3}(X, Y, \tau, T)+\epsilon^{4} \mathrm{e}^{i \psi} I_{1}(X, Y, \tau, T) \\
& +\epsilon^{4} e^{2 i \psi} I_{2}(X, Y, \tau, T)+\epsilon^{4} e^{3 i \psi} I_{3}(X, Y, \tau, T)+\epsilon^{4} \mathrm{e}^{4 i \psi} I_{4}(X, Y, \tau, T) \\
& +\epsilon^{5} \mathrm{e}^{i \psi} J_{1}(X, Y, \tau, T)+\cdots+c . c .,
\end{aligned}
$$

where the phase, $\psi$, is given by $k m+l n+\omega t$. We substitute the ansatz (2.18) into the governing equations (2.14) and equate coefficients of each harmonic frequency at each order of $\epsilon$. This yields the following equations

$\mathcal{O}\left(\epsilon \mathrm{e}^{i \psi}\right)$

$$
\omega^{2} F=4 \sin ^{2}\left(\frac{1}{2} k\right) F+4 \sin ^{2}\left(\frac{1}{2} l\right) F,
$$

$\mathcal{O}\left(\epsilon^{2} \mathrm{e}^{i \psi}\right)$

$$
\omega F_{\tau}=F_{X} \sin k+F_{Y} \sin l,
$$

$\mathcal{O}\left(\epsilon^{2} \mathrm{e}^{2 i \psi}\right)$

$$
\omega^{2} G_{2}=\left[\sin ^{2} k+\sin ^{2} l\right] G_{2}+a\left[\sin ^{2} k+\sin ^{2} l\right] F^{2},
$$


$\mathcal{O}\left(\epsilon^{3} \mathrm{e}^{i \psi}\right)$

$$
\begin{aligned}
2 i \omega F_{T}+F_{\tau \tau}= & F_{X X} \cos k+F_{Y Y} \cos l \\
& -8 a\left[\sin ^{2}\left(\frac{1}{2} k\right)+\sin ^{2}\left(\frac{1}{2} l\right)\right]\left[F\left(G_{0}+\bar{G}_{0}\right)+\bar{F} G_{2}\right] \\
& -12 b\left[\sin ^{2}\left(\frac{1}{2} k\right)+\sin ^{2}\left(\frac{1}{2} l\right)\right]|F|^{2} F
\end{aligned}
$$

$\mathcal{O}\left(\epsilon^{4} \mathrm{e}^{0}\right)$

$$
G_{0 \tau \tau}=G_{0 X X}+G_{0 Y Y}+a\left(|F|^{2}\right)_{X X}+a\left(|F|^{2}\right)_{Y Y} .
$$

A quick inspection of equations (2.19)-(2.23) reveals that (2.19) is the dispersion relation for the system (2.14). Since we are interested only in solutions for which $F \neq 0, F$ can be cancelled from (2.19), giving the dispersion relation for the system

$$
\omega^{2}=4 \sin ^{2}\left(\frac{1}{2} k\right)+4 \sin ^{2}\left(\frac{1}{2} l\right) .
$$

Equation (2.20) gives the relationship between the temporal and spatial derivatives of $F$, and from it we deduce that $F$ is a travelling wave of the form

$$
F(X, Y, \tau, T) \equiv F(Z, W, T)
$$

where $Z=X-u \tau, W=Y-v \tau$. The horizontal and vertical velocities of the wavepacket $F$ are $u=-(\sin k) / \omega$ and $v=-(\sin l) / \omega$ respectively; these definitions, together with (2.24) enable the elimination of $G_{1}$ from (2.22). The angle of propagation of the envelope $(\Psi)$ is given by $\tan ^{-1}(v / u)=\tan ^{-1}(\sin k / \sin l)$. We expect $(2.22)$ to reduce to a version of the nonlinear Schrödinger equation in $F$ (as occurred in the one-dimensional FPU analysis). Before this can be done, the quantities $G_{0}$ and $G_{2}$ must be found in terms of $F$. The former is found easily from the algebraic equation (2.21). However, in general, the partial differential equation (2.23) cannot be solved for $G_{0}$, save for two special cases. These two cases are detailed in the following sections.

\subsection{The lattice with a symmetric potential}

By a symmetric potential, we mean one for which $\mathcal{F}^{\prime}(-Q)=-\mathcal{F}^{\prime}(Q)$, that is, one for which $\mathcal{F}^{\prime}(Q)$ has odd symmetry and $\mathcal{F}(Q)$ is even. This corresponds to $a=c=0$ in (2.12) and (2.14), and $\mathcal{F}(Q)$ has only fourth-order and sixth-order nonlinear terms in addition to the harmonic term. In this case, it is immediately clear that $G_{0}=G_{2}=0$, since a vibration controlled by a symmetric potential cannot contain any even harmonics.

It remains to find $F_{\tau \tau}$ in terms of $F_{Z Z}, F_{Z W}$ and $F_{W W}$ using (2.25), and then substituting into (2.22) gives

$$
2 i \omega F_{T}+\left[u^{2}-\cos k\right] F_{Z Z}+\left[v^{2}-\cos l\right] F_{W W}+2 u v F_{Z W}+3 b \omega^{2}|F|^{2} F=0 .
$$

Equation (2.26) is a two-dimensional NLS equation with cubic nonlinearity. The presence of mixed derivative terms of the form $F_{Z W}$ in (2.26) complicates matters. 
The equation can be reduced to a standard form by eliminating the mixed term $F_{Z W}$. This is done by introducing the transformation of variables

$$
\begin{aligned}
& \xi=\frac{Z}{\sqrt{u^{2}-\cos k}} \text { and } \\
& \eta=\frac{-u v Z+\left[u^{2}-\cos k\right] W}{\sqrt{\left(u^{2}-\cos k\right)\left[\cos k \cos l-u^{2} \cos l-v^{2} \cos k\right]}},
\end{aligned}
$$

which maps (2.26) to the equation

$$
2 \omega i F_{T}+\nabla^{2} F+3 b \omega^{2}|F|^{2} F=0,
$$

where the Laplacian operator is defined by $\nabla^{2} F=F_{\xi \xi}+F_{\eta \eta}$. Equation (2.28) has the same form as (3.1) with $D=1 / 2 \omega$ and $B=3 b \omega / 2$. Since the differential operator in (2.28) is isotropic, we will seek radially symmetric solutions of this equation of the form $F=\mathrm{e}^{i \lambda T} \phi(r)$ where $r^{2}=\xi^{2}+\eta^{2}$. Approximations to $\phi(r)$ can be generated using the method outlined in the appendix.

\subsection{Determining the domain of ellipticity}

The two-dimensional NLS equation (2.26) admits different types of solution depending on whether the equation is elliptic or hyperbolic. By definition, the equation is elliptic when $u^{2} v^{2}<\left(u^{2}-\cos k\right)\left(v^{2}-\cos l\right)$. We aim to determine the region $\mathcal{D}$ of $(k, l)$ parameter space (which is the two-torus $T^{2}=[0,2 \pi] \times[0,2 \pi]$ ) in which this inequality is satisfied. Substituting for $u, v$ and $\omega$ from (2.24) and (2.25), the condition for ellipticity is

$$
\sin ^{4}\left(\frac{1}{2} k\right)\left[1-2 \sin ^{2}\left(\frac{1}{2} l\right)\right]+\sin ^{4}\left(\frac{1}{2} l\right)\left[1-2 \sin ^{2}\left(\frac{1}{2} k\right)\right]<0 .
$$

Since the function on the left hand side of (2.29) is symmetric in $k$ and $l$ about $\pi$, it is only necessary to consider one-quarter of the two-torus $T^{2}$, namely the subspace $[0, \pi] \times[0, \pi]$.

Defining $\theta$ and $\sigma$ by $\theta=\sin ^{2}\left(\frac{1}{2} k\right)$ and $\sigma=\sin ^{2}\left(\frac{1}{2} l\right)$, then $0 \leq \theta, \sigma \leq 1$ and the condition for ellipticity (2.29) becomes

$$
\theta^{2}(1-2 \sigma)+\sigma^{2}(1-2 \theta)<0 .
$$

Substituting $\theta=\rho \cos \zeta, \sigma=\rho \sin \zeta$, into (2.30) leads to the inequality

$$
\rho<\frac{1}{2 \cos \zeta \sin \zeta(\cos \zeta+\sin \zeta)} .
$$

It is straightforward to show that $\pi / 8 \leq \zeta \leq 3 \pi / 8$, (since $0 \leq \theta, \sigma \leq 1$ ). The inequality (2.31) defines in parametric form the curve in $(\rho, \zeta)$-space which constitutes the boundary of the region where the equation (2.26) is elliptic; this boundary is shown in Figure 2.3(a). Reverting back to the variables $\theta$ and $\sigma$, the boundary of the region of ellipticity of $(2.26)$ in $(\theta, \sigma)$-space can be found; this is the shaded area shown in Figure 2.3(b) and Figure 2.3(c) shows the domain of ellipticity in $(k, l)$ space. 


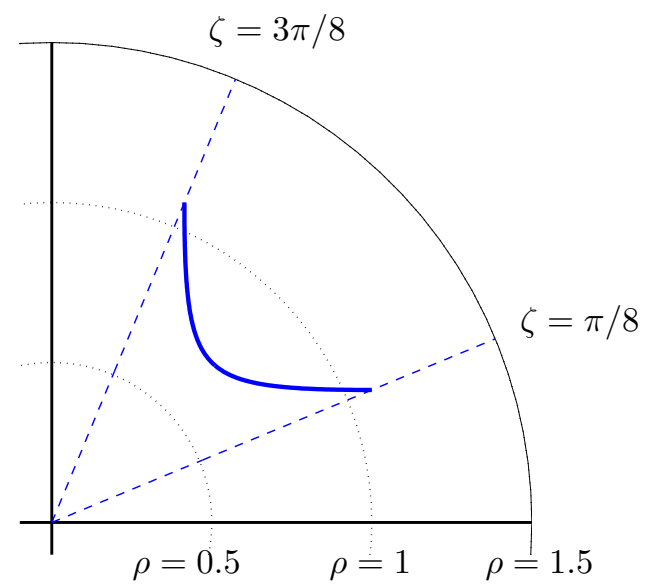

(a) Boundary of region of ellipticity in $(\rho, \zeta)$-space

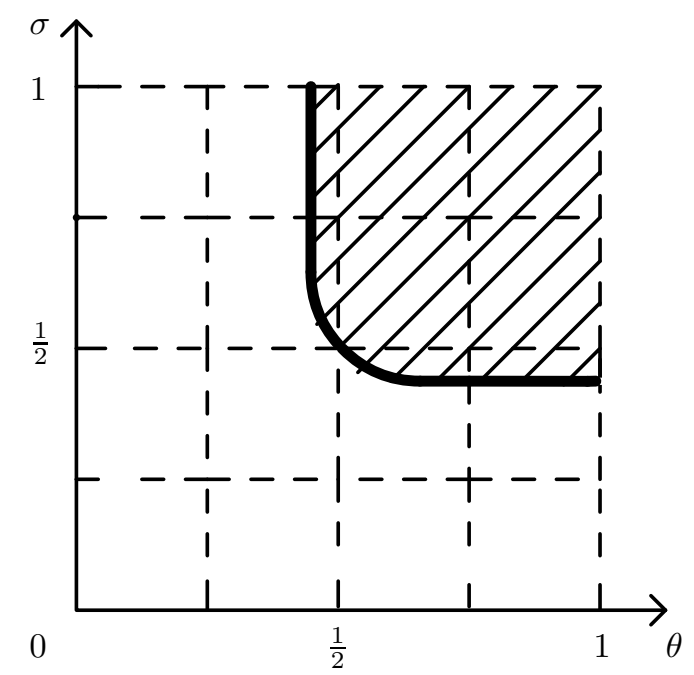

(b) Region of ellipticity shown in $(\theta, \sigma)$ space

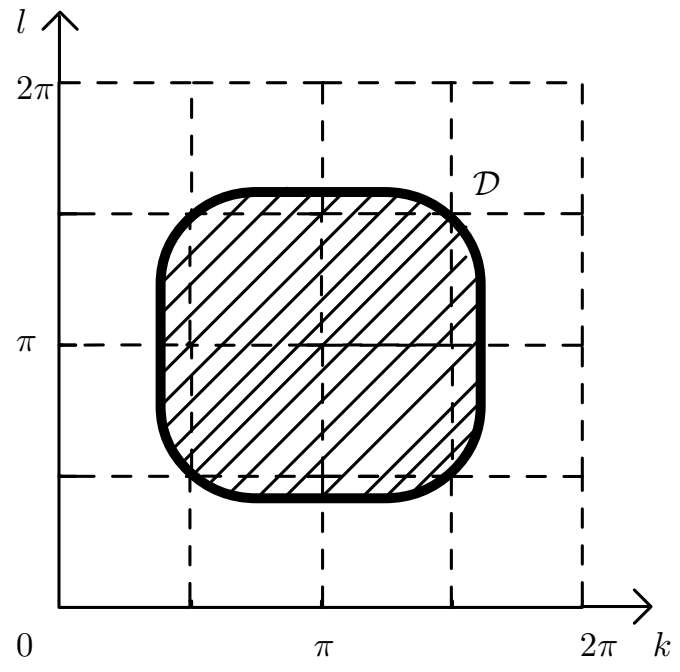

(c) Domain $\mathcal{D}$ in $(k, l)$-space

Figure 2.3. The domain $\mathcal{D}$ in which the NLS equation (2.26) is elliptic.

\subsection{The lattice with an asymmetric potential}

When an asymmetric potential is considered, equation (2.22) can be reduced to an NLS equation for $F$ for the special case $k=l=\pi$. In this case, the partial differential equation (2.23) can be solved to give $G_{0}$ explicitly in terms of $F$. Since $G_{0}$ is a higher order correction term to the leading order quantity $F$, it also travels at the same velocity as $F$, that is, $G_{0}(X, Y, \tau, T) \equiv G_{0}(Z, W, T)$. Rewriting (2.23) in terms of the variables 
$Z$ and $W$ yields

$$
\left(u^{2}-1\right) G_{0 Z Z}+\left(v^{2}-1\right) G_{0 W W}+2 u v G_{0 Z W}=a\left(|F|^{2}\right)_{Z Z}+a\left(|F|^{2}\right)_{W W} \cdot(2 .
$$

Since $k=l=\pi$ implies that $v=c=0$, we have $\nabla^{2} G_{0}=-a \nabla^{2}|F|^{2}$ and hence $G_{0}=-a|F|^{2}$, where the operator $\nabla^{2}$ defined by $\nabla_{(Z, W)}^{2} \equiv \partial_{Z Z}+\partial_{W W}$ is equivalent to $\nabla_{(X, Y)}^{2} \equiv \partial_{X X}+\partial_{Y Y}$. Clearly $G_{2}=0$ from (2.21). Substituting for $G_{0}$ in (2.22) gives

$$
i F_{T}+\frac{\sqrt{2}}{8} \nabla^{2} F+\sqrt{2}\left(3 b-4 a^{2}\right)|F|^{2} F=0,
$$

which is a two-dimensional NLS equation in $F(X, Y, T)$. As with (2.28), we will be seeking radially symmetric solutions of the form $F=\mathrm{e}^{i \lambda T} \phi(r)$ where $r^{2}=X^{2}+Y^{2}$.

\section{Townes solitons and stabilisation by higher-order terms}

\subsection{Townes solitons}

In Sections 2.4 and 2.6, we have seen that it is possible to reduce the lattice equations (2.14) to a two-dimensional NLS equation in $F$, where $F$ determines the form of the envelope of a breather solution. At this point, one might consider proceeding as in the one-dimensional case (see for example, Butt \& Wattis [30]) and using a soliton solution for $F$ to substitute back into the ansatz (2.18). This in turn would give a leading order analytic form for the charge $Q_{m, n}$. However, this approach cannot be used directly for the two-dimensional case because the two-dimensional cubic nonlinear Schrödinger equation does not support stable soliton solutions. It is worth reviewing briefly the scalar two-dimensional nonlinear Schrödinger equation and generalisations of it.

Firstly, we summarise the relevant results on the phenomenon of blow-up or selffocusing of solutions $F(\mathbf{x}, T)\left(\mathbf{x} \in \mathbb{R}^{d}, T \in[0, \infty)\right.$ and $\left.F(\mathbf{x}, T) \in \mathbb{C}\right)$ of the scalar $d$-dimensional NLS equation

$$
i F_{T}+D \nabla^{2} F+B|F|^{2} F=0 .
$$

For an in-depth account of the NLS equation and self-focusing, see Sulem \& Sulem [32]. There are two distinct cases of (3.1), namely the anomalous dispersion regime in which case $D B>0$, also known as the focusing NLS equation; and the normal dispersion regime where $D B<0$, also known as the defocusing NLS equation. It is well known that all solutions of the defocusing NLS equation exist globally, that is, for all $T \in[0, \infty)$. However, in this case, it is also known that (3.1) admits no localised solutions. In particular, the defocusing NLS equation does not admit soliton solutions. Since we are interested only in soliton solutions of (3.1), we will concern ourselves primarily with the focusing NLS equation (that is, the anomalously dispersive regime for which $B D>0$ ).

In any dimension $d \geq 2$, solutions of the focusing NLS equation with smooth initial conditions can become singular in a finite time. This is known as blow-up, or selffocusing. In physical terms, the amplitude of a wavepacket becomes infinite at a single point called the focus, and this is accompanied by radial contraction of the wavepacket. Of central importance is the establishment of conditions under which the formation 
of a singularity either occurs or is prevented. Some useful theorems regarding global solutions (those which exist for all $T \in[0, \infty)$ ) and local solutions (existence only for a finite time interval $[0, T)$, for some $T>0$ ) are presented by Strauss [33]. It is well known that for $d=1$, the competing effects of nonlinearity and dispersion in (3.1) may balance each other, giving rise to long-lived solitonic structures (Remoissenet [34]). This is not so for systems with $d \geq 2$. We will be concerned only with $d=2$, the so-called critical dimension, in which there exists a very fine balance between focusing and dispersive effects.

For systems of any dimension $d$, there are two invariants associated with solutions of (3.1), namely the Hamiltonian $\mathcal{H}$ and the power $\mathcal{N}$, given respectively by

$$
\mathcal{H}=\iint\left\{D|\nabla F|^{2}-\frac{1}{2} B|F|^{4}\right\} \mathrm{d}^{2} \mathbf{x} \quad \text { and } \quad \mathcal{N}=\iint|F|^{2} \mathrm{~d}^{2} \mathbf{x}
$$

Using the variance identity, the "uncertainty principle" and the invariance of $\mathcal{H}$, it can be shown that $\mathcal{H}<0$ is a sufficient condition for blow-up (see Sulem \& Sulem [32] for further details). For the two-dimensional case, a necessary condition for blow-up is that the initial power $\mathcal{N}_{0}=\mathcal{N}(0)$ must exceed some critical threshold $\mathcal{N}_{c}$, that is, $\mathcal{N}_{0} \geq \mathcal{N}_{c}$, where $\mathcal{N}_{c}$ is a number that depends only upon the dimension. Typically, for $B=D=1$, $\mathcal{N}_{c} \approx 1.862$ (Fibich \& Papanicolaou [35]). The focusing NLS equation in $\mathbb{R}^{2}(3.1)$ supports a one-parameter family of soliton solutions of the form $F(\mathbf{x}, T)=e^{i \lambda T} R_{0}(r)$, where $r=|\mathbf{x}|$, known as Townes solitons (see Chiao et al. [31]). Townes solitons are highly unstable since they have $\mathcal{H}=0$ and $\mathcal{N}=\mathcal{N}_{c}$. Clearly a tiny perturbation could give $\mathcal{H}<0$ or $\mathcal{N}>\mathcal{N}_{c}$, leading to blow-up (or $\mathcal{H}>0$ and $\mathcal{N}<\mathcal{N}_{c}$ leading to decay).

\subsection{Stable soliton solutions of generalised NLS equations}

The cubic NLS equation (3.1) exhibits behaviour that is impossible in the context of our spatially discrete system: in a discrete system, the focusing of all the system's energy at a single point will not lead to blow-up, in a discrete system the amplitude would not diverge. In Section 4 we present numerical simulations which suggest that long-lived breather modes are supported by the scalar two-dimensional Fermi-Pasta-Ulam lattice. We therefore conclude that the cubic two-dimensional NLS equation does not correctly capture the actual dynamics of the two-dimensional Fermi-Pasta-Ulam lattice.

In applications, the phenomenon of blow-up is prevented by for instance, dissipation, or higher order effects (see Rasmussen \& Rypdal [36] for a useful review). When blow-up arises in an evolution equation, it indicates a failure of the model of the system under consideration, or equivalently, that the assumptions made in deriving the model are not valid. Of direct relevance to our work, as suggested by Kivshar \& Pelinovsky [37], in quasi-continuum models such as ours, where discreteness is approximated by a continuous variable, the effects of higher-order dispersion play a vital role, and therefore must be included.

In particular, we are interested in collapse arresting, whereby the blowing-up of a localised solution may be prevented by some extra mechanism. Of course, this in itself is not enough to guarantee the existence of robust soliton solutions, since it does 
not preclude the possibility of decay through dispersion or dissipation. Hence it is also important to check for soliton regularisation or soliton management, which ensures robustness follows collapse inhibition. In fact, various mechanisms for suppression of wave collapse have now been found (see for example, the work of Schjødt-Eriksen et al. [38] or Abdullaev et al. [39]). Consequently, it is known that unstable Townes soliton solutions of the cubic scalar two-dimensional NLS equation may be stabilised through a variety of mechanisms. We concern ourselves only with those mechanisms which are relevant and applicable to lattice models. For instance, it was shown by Karpman [40] that an additional higher order dispersion term can have a stabilising effect. Specifically, the generalised two-dimensional NLS equation with higher order dispersion

$$
i F_{T}+D \nabla^{2} F+B|F|^{2} F+P \nabla^{4} F=0,
$$

can support stable soliton solutions when $P D<0$. Of greatest relevance to us is a result of Davydova et al. [41], whom establish the existence of stable solitons for a generalised two-dimensional NLS equation which incorporates higher order dispersion and an additional quintic nonlinear term, namely

$$
i F_{T}+D \nabla^{2} F+B|F|^{2} F+P \nabla^{4} F+K|F|^{4} F=0 .
$$

The possibility of stable soliton solutions is investigated in both the anomalous $(B D>0)$ and normal $(B D<0)$ dispersive regimes. It is found that stable soliton solutions exist in both regimes.

Both of these results are established using a variational method described more fully by Kuznetsov et al. [42]. In this method, one seeks localised standing wave solutions of the form $e^{i \lambda T} \phi(\mathbf{x})(\lambda>0)$ of the NLS equation in question. By defining an action integral $\mathcal{S}(\phi)=\mathcal{H}(\phi)+\mathcal{N}(\phi)$ ( $\mathcal{H}$ is the Hamiltonian for the relevant NLS equation), it is shown that soliton solutions of the NLS equation correspond to extrema (actually expected to be minima) of this action integral. Minima of $\mathcal{S}$ are then identified, using a procedure developed by Derrick [43]. Once the existence of soliton solutions has been proved, their stability properties must then be established. Again, as described by Kuznetsov et al. [42], the functional $\mathcal{S}(F)=\mathcal{H}(F)+\mathcal{N}(F)$ constitutes an appropriate Lyapunov function (up to some constant quantity rendering it positive definite). Soliton stability follows upon applying Lyapunov's theorem.

Further insight can be gained by considering a virial relation for (3.4). One defines an effective "beam" radius $r_{\text {eff by }}$

$$
r_{\text {eff }}^{2}(T)=\frac{1}{\mathcal{N}} \iint r^{2}|F(\mathbf{x}, T)|^{2} \mathrm{~d}^{2} \mathbf{x}
$$

Differentiating (3.5) with respect to time reveals whether the radius of a soliton grows without limit (soliton dispersal) or decreases to zero (blow-up) as time evolves. In particular, the effect of the individual parameters on soliton stability becomes clear.

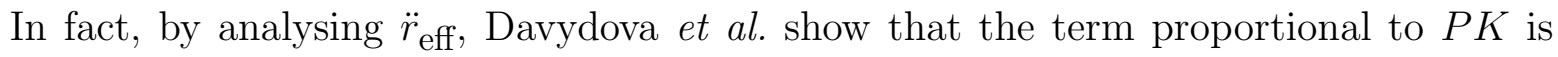
the dominant term, and provided $P K>0$, this corresponds to a defocusing term. In other words, if $P K>0$, the dominant term provides a "repulsive force" which prevents 
collapse in both regimes. Hence stable soliton solutions can be found in the parameter regime $P K>0$ for both anomalous and normal cases.

\subsection{Higher order asymptotic analysis for stationary breathers}

In the light of comments made in Section 3.2, we extend our analysis of (2.14) to fifthorder and reduce the lattice equations (2.14) to a generalised nonlinear Schrödinger equation which also includes higher order terms. The principal task is to determine whether the new terms are stabilising, and in particular, whether the generalised NLS equation admits stable soliton solutions. For the general case of moving breathers, the equations are extremely complicated, hence we simplify matters by considering only those cases we are able to solve for, namely, stationary breathers $(k=l=\pi$ implying $u=v=0)$, and symmetric potentials $(a=c=0)$ as in Section 2.4.

For stationary breathers, only one extra timescale is used, $T=\epsilon^{2} t$, and we consider specific wavenumbers $k=\pi$ and $l=\pi$. In this case, for a symmetric potential $(a=c=0)$, the ansatz (2.18) takes the much simplified form

$$
Q_{m, n}(t)=\epsilon \mathrm{e}^{i \psi} F(X, Y, T)+\epsilon^{3} \mathrm{e}^{3 i \psi} H_{3}(X, Y, T)+\cdots+\text { c.c. },
$$

where $\exp (i \psi)=\exp (i \omega t+i \pi m+i \pi n)=(-1)^{m+n} \exp (i \omega t)$. Substituting the ansatz (3.6) into equations (2.14) yields the following set of equations.

$\mathcal{O}\left(\epsilon \mathrm{e}^{i \psi}\right)$ :

$$
\omega^{2}=8 \Rightarrow \omega=2 \sqrt{2},
$$

$\mathcal{O}\left(\epsilon^{3} \mathrm{e}^{i \psi}\right)$

$$
2 i \omega F_{T}+F_{X X}+F_{Y Y}+24 b|F|^{2} F=0
$$

$\mathcal{O}\left(\epsilon^{3} \mathrm{e}^{3 i \psi}\right)$

$$
9 \omega^{2} H_{3}=8 H_{3}+8 b F^{3} \Rightarrow H_{3}=\frac{1}{8} b F^{3}
$$

$\mathcal{O}\left(\epsilon^{5} \mathrm{e}^{i \psi}\right)$

$$
\begin{aligned}
F_{T T}= & -\frac{1}{12} F_{X X X X}-\frac{1}{12} F_{Y Y Y Y}-80 d F^{3} \bar{F}^{2}-24 b \bar{F}^{2} H_{3} \\
& -3 b\left[F^{2} \bar{F}_{X X}+2 F \bar{F} F_{X X}+4 F F_{X} \bar{F}_{X}+2 \bar{F} F_{X}^{2}\right] \\
& -3 b\left[F^{2} \bar{F}_{Y Y}+2 F \bar{F} F_{Y Y}+4 F F_{Y} \bar{F}_{Y}+2 \bar{F} F_{Y}^{2}\right] .
\end{aligned}
$$

We aim to combine the higher order terms of (3.10) with the cubic two-dimensional NLS equation (3.8). Firstly, the term $F_{T T}$ in (3.10) is eliminated by differentiating (3.8) with respect to $T$ and substituting. Once an expression for $F_{T T}$ is found, the combination (3.8) and (3.10) becomes

$$
\begin{aligned}
& 4 \sqrt{2} i F_{T}+\nabla^{2} F+24 b|F|^{2} F+\epsilon^{2}\left[\frac{5}{96} \nabla^{4} F-\left(51 b^{2}-80 d\right)|F|^{4} F\right] \\
& -\frac{1}{6} \epsilon^{2} F_{X X Y Y}+\frac{9}{4} b \epsilon^{2} \nabla^{2}\left(|F|^{2} F\right)-\frac{3}{4} b \epsilon^{2}\left(2|F|^{2} \nabla^{2} F+F^{2} \nabla^{2} \bar{F}\right)=0 .
\end{aligned}
$$

In other words, the lattice equations (2.14) reduce to the generalised two-dimensional NLS equation (3.11) for the envelope $F$. To our knowledge, this equation has not been 
studied in the literature, and therefore we are unable to say conclusively whether it supports stable soliton solutions. We note that the first line of (3.11) is identical to the two-dimensional NLS equation (3.4) studied by Davydova et al. [41], and includes known stabilising terms. Anomalous dispersion corresponds to $b>0$ and the condition $P K>0$ reduces to $80 d>51 b^{2}$. The effects of the other correction terms remain unknown. At the time of writing, we have been unable to find a variational form for (3.11), hence the methods outlined by Kuznetsov et al. [42] and Davydova et al. [41] cannot be applied directly. In fact, given the complexity of (3.11), a more suitable approach might be to apply the modulation theory for perturbed NLS equations, covered in great depth by Fibich \& Papanicolaou [44, 35]. This approach reduces a perturbed NLS equation to a simpler set of equations in which one can (in principle) determine the effect of small perturbations upon focusing. In particular, Fibich \& Papanicolaou [44, 35] derive results on the focusing properties of classes of perturbative terms. Depending upon the specific perturbation terms present, one of several different outcomes can result. For instance, blow-up may occur, or on the other hand, it may be prevented completely. In addition, one might find a solitary wave solution which undergoes small oscillations in radial width. Analysis of (3.11) using one of the above methods is the subject of future work. For now, we rely on Davydova's criteria for soliton existence, and supplement our theoretical work with numerical simulations of the lattice equations (2.14).

\section{Numerical results}

In this section, the equations governing charge $Q_{m, n}$ in the lattice, (2.14), are solved numerically. Equations (2.14) comprise an infinite set of coupled second-order nonlinear ordinary differential equations. In order to solve these numerically, we consider these equations defined on a square lattice of $N \times N$ sites. (To allow for computation within reasonable times, typically we consider lattices for which $N \leq 50$ ). The numerical algorithm used to solve the equations is based on a fourth-order Runge-Kutta scheme, implemented by MATLAB. Firstly, the system of $N^{2}$ second-order ordinary differential equations is converted to an equivalent system of $2 N^{2}$ first-order equations. The variable $\mathcal{P}_{m, n}$ is introduced, where $\mathcal{P}_{m, n}=\mathrm{d} Q_{m, n} / \mathrm{d} t$ (note that this definition differs from (2.16)). Equations (2.14) are thus equivalent to

$$
\begin{aligned}
& \frac{\mathrm{d} Q_{m, n}}{\mathrm{~d} t}=\mathcal{P}_{m, n}, \quad \text { and } \\
& \begin{aligned}
\frac{\mathrm{d} \mathcal{P}_{m, n}}{\mathrm{~d} t}=\left(\delta_{m}^{2}+\delta_{n}^{2}\right)\left[Q_{m, n}+a Q_{m, n}^{2}+b Q_{m, n}^{3}+c Q_{m, n}^{4}+d Q_{m, n}^{5}\right], \\
\\
\quad m, n=1,2, \ldots, N .
\end{aligned}
\end{aligned}
$$

\subsection{Boundary conditions}

Selecting the site $(1,1)$ to lie at the lattice site at the bottom left hand corner of the square arrangement in Figure 4.1, the remaining lattice sites are labelled with a coordinate pair $(m, n)$ denoting their relative horizontal and vertical positions. That 
is, site $(m, n)$ denotes the site which lies in the $m^{\text {th }}$ column across and in the $n^{\text {th }}$ row up. The charge stored on each capacitor depends on the charge stored on the capacitors located at the four nearest neighbouring sites, namely, the capacitors at either side and above and below (looking at the lattice from above, see Figure 4.1). Equations (4.1) are defined at interior lattice sites, but sites along the boundary are missing at least one neighbour (two neighbours are missing for each of the corner sites). We impose periodic boundary conditions for the lattice such that it is effectively "wrapped around" in both the horizontal and vertical directions, so that the lattice is in effect a twotorus. The physical significance of this is that a disturbance moving horizontally to the right (left) within the lattice disappears from the far right (left) hand edge of the lattice and reemerges from the left (right) hand edge, with similar properties in the vertical direction. In terms of fictitious points along the lattice boundary we impose the following boundary conditions (illustrated in Figure 4.1)

$$
\begin{array}{ll}
\mathcal{P}_{N+1, n}=\mathcal{P}_{1, n}, & \mathcal{P}_{m, N+1}=\mathcal{P}_{m, 1}, \quad \mathcal{P}_{0, n}=\mathcal{P}_{N, n}, \quad \mathcal{P}_{m, 0}=\mathcal{P}_{m, N}, \\
Q_{N+1, n}=Q_{1, n}, & Q_{m, N+1}=Q_{m, 1}, \quad Q_{0, n}=Q_{N, n}, \quad Q_{m, 0}=Q_{m, N} .
\end{array}
$$

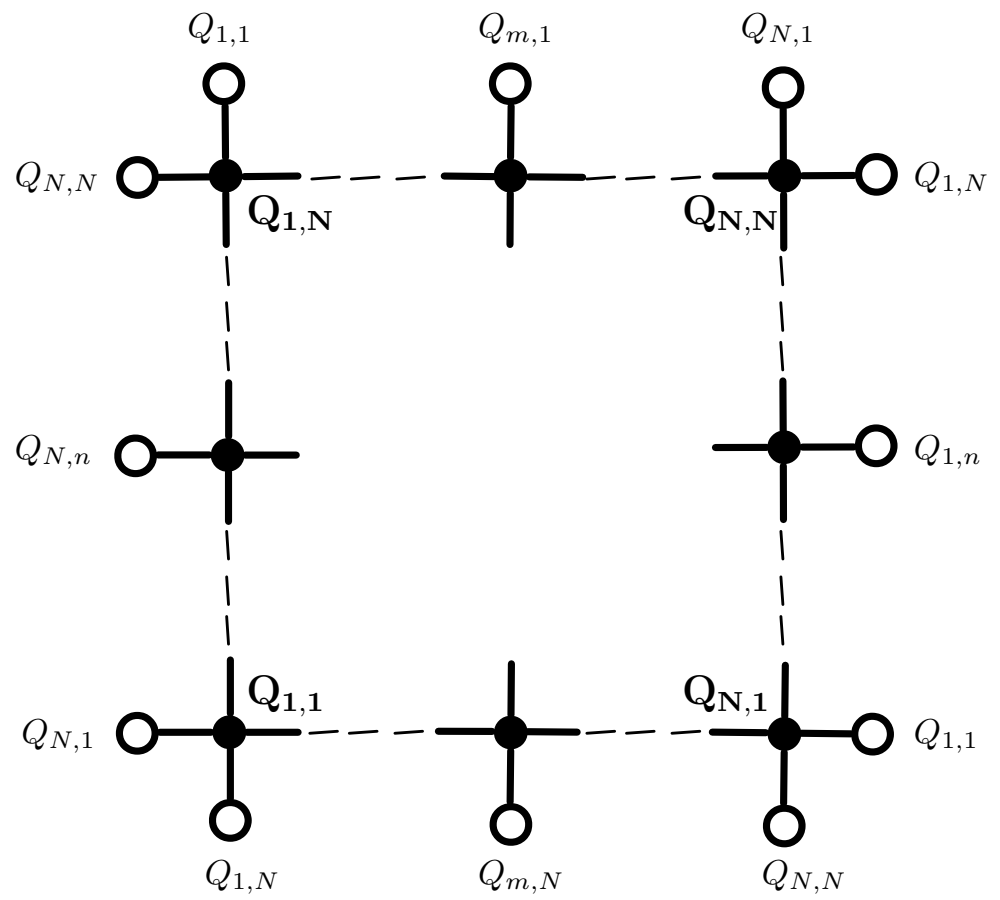

Figure 4.1. Boundary conditions for the two-dimensional ETL

\subsection{Breather energy}

In this section, we discuss the calculation of the energy of breathers in the twodimensional ETL. Since the 2D ETL is a lossless network, the total electrical energy is conserved. For small currents and small voltages, the leading order energy in one unit 
of the lattice is

$$
e_{m, n}^{(0)} \sim \frac{1}{2} C_{0} V_{m, n}^{2}+\frac{1}{4} L\left(I_{m, n}^{2}+J_{m, n}^{2}+I_{m-1, n}^{2}+J_{m, n-1}^{2}\right) .
$$

Thus the leading order expression for the total electrical energy in the lattice is (see Figure 2.2)

$$
E^{(0)} \sim \sum_{m, n} e_{m, n}^{(0)}=\sum_{m, n} \frac{1}{2} C_{0} V_{m, n}^{2}+\frac{1}{2} L\left(I_{m, n}^{2}+J_{m, n}^{2}\right) .
$$

We present a leading order asymptotic estimate of the breather energy $E^{(0)}(4.4)$ using the leading order expression for $Q_{m, n}$ (A.8). Two separate cases are considered. For both, since we aim to determine only a leading order estimate, it follows from (2.12) that $V_{m, n} \sim Q_{m, n} / C_{0}$. Hence, the first term of the summand in (4.4) is simply $Q_{m, n}^{2} /\left(2 C_{0}\right)$. The currents $I_{m, n}$ and $J_{m, n}$ may also be found in terms of $V_{m, n}$ and $Q_{m, n}$. By (2.5)(2.6), since $L C_{0}=1$ and to leading order $Q=C_{0} V$, we have $\dot{I}_{m, n}=-\left(Q_{m+1, n}-Q_{m, n}\right)$ and $\dot{J}_{m, n}=-\left(Q_{m, n+1}-Q_{m, n}\right)$. Hence by $(2.16)$ with $\mathcal{F}(Q)=Q^{2} / 2$, we obtain $I_{m, n}=P_{m+1, n}-P_{m, n}$ and $J_{m, n}=P_{m, n+1}-P_{m, n}$, and so $L I_{m, n}^{2}=\left(1 / C_{0}\right)\left(P_{m+1, n}-P_{m, n}\right)^{2}$ and $L J_{m, n}^{2}=\left(1 / C_{0}\right)\left(P_{m, n+1}-P_{m, n}\right)^{2}$. Thus $E^{(0)}=\left(1 / C_{0}\right) \widetilde{H}$ with $\widetilde{H}$ as given by $(2.15)$. The details of the evaluation of $E^{(0)}$ are slightly different for stationary and moving breathers; we consider each in turn below.

4.2.1. Stationary breathers In this case, $k=l=\pi$, and from (A.8), the leading order from of the breather is

$$
Q_{m, n} \sim 2 \epsilon \alpha \cos \Theta \operatorname{sech}(\beta r),
$$

where $\omega=2 \sqrt{2}$ is the leading order breather frequency, $\Omega=\omega+\epsilon^{2} \lambda$ is the breather frequency including the first correction term, $\lambda$ parameterises the amplitude of the breather (see Appendix A for more details), $\Theta=\pi m+\pi n+\Omega t$ describes the phase of the linear wave, and $r=\sqrt{X^{2}+Y^{2}}=\epsilon \sqrt{m^{2}+n^{2}}$ is a scaled distance from the centre of the breather. Since $V_{m, n} \sim Q_{m, n} / C_{0}$, the equation for the current $I_{m, n}(2.5)$ becomes

$$
Q_{m+1, n}-Q_{m, n} \sim-L C_{0} \frac{\mathrm{d} I_{m, n}}{\mathrm{~d} t}=-\frac{\mathrm{d} I_{m, n}}{\mathrm{~d} t} .
$$

Substituting the expression for $Q_{m, n}$ (4.5) into the right hand side of (4.6) and integrating with respect to $t$ gives the current

$$
I_{m, n} \sim-\frac{4 \epsilon \alpha}{\omega} \sin \Theta \operatorname{sech}(\beta r),
$$

where we have taken the constant of integration to be zero, and $\Omega \sim \omega$ to leading order. In a similar manner, taking (2.6) and integrating gives

$$
J_{m, n} \sim-\frac{4 \epsilon \alpha}{\omega} \sin \Theta \operatorname{sech}(\beta r),
$$

Hence overall, the energy $E^{(0)}$ in (4.4) is given by

$$
E^{(0)} \sim \sum_{m, n} \frac{2 \epsilon^{2} \alpha^{2}}{C_{0}} \cos ^{2} \Theta \operatorname{sech}^{2}(\beta r)+\frac{16 L \epsilon^{2} \alpha^{2}}{\omega^{2}} \sin ^{2} \Theta \operatorname{sech}^{2}(\beta r) .
$$


Recall that $\omega^{2}=8$, and also that $L C_{0}=1$ or equivalently, $L=1 / C_{0}$. The sum in (4.9) therefore simplifies considerably and becomes

$$
E^{(0)} \sim \sum_{m, n} \frac{2 \epsilon^{2} \alpha^{2}}{C_{0}} \operatorname{sech}^{2}(\beta r) .
$$

Since the variables $X=\epsilon m$ and $Y=\epsilon n$ vary slowly with $m, n$, so does $r$ and we can justifiably replace the sum in $(4.10)$ by an integral. Hence, the energy $E^{(0)}$ is given by

$$
E^{(0)} \sim \frac{2 \alpha^{2}}{C_{0}} \iint \operatorname{sech}^{2}\left(\beta \sqrt{X^{2}+Y^{2}}\right) \mathrm{d} X \mathrm{~d} Y
$$

Upon calculating the integral in (4.11), we find that

$$
E^{(0)} \sim \frac{4 \pi \log 2}{C_{0}} \frac{\alpha^{2}}{\beta^{2}}=\frac{8 \pi D \log 2(2 \log 2+1)}{B C_{0}(4 \log 2-1)},
$$

after substituting for $\alpha$ and $\beta$ from (A.5). This can be approximated by $E^{(0)} \approx$ $23.452 D / B C_{0}$. In the general case of the asymmetric potential for which stationary breathers have been found, we compare (2.33) with (A.1) we find $D=1 / 4 \sqrt{2}$ and $B=\sqrt{2}\left(3 b-4 a^{2}\right)$, thus $E^{(0)} \approx 2.935 /\left(3 b-4 a^{2}\right) C_{0}$. Note that this leading-order expression is independent of breather amplitude $\lambda$, illustrating the threshold energy requirement for breathers in two-dimensional systems; that is, no matter how small the amplitude of a breather $(2 \epsilon \alpha)$, the energy required to create it is an $\mathcal{O}(1)$ quantity.

4.2.2. Moving breathers In this case, the leading order form of the breather is given by (A.8). Again, the first term of the summand in (4.4) is $Q_{m, n}^{2} /\left(2 C_{0}\right)$, and the current $I_{m, n}$ is given by (4.6). The expression for $Q_{m, n}$ (A.8) is substituted into (4.6), only this time, the left hand side is not easily integrated with respect to time. To overcome this, we note that in (A.8), the variable $r$ varies slowly in time compared to the phase of the oscillatory component $\Phi=k m+l n+\Omega t$. Integration by parts of (4.6) using (4.5) $\left(\int f^{\prime}(t) g(\epsilon t) \mathrm{d} t=[f(t) g(\epsilon t)]-\epsilon \int f(t) g^{\prime}(\epsilon t) \mathrm{d} t\right)$ then implies that to leading order we have

$$
I_{m, n} \sim-\frac{2 \epsilon \alpha}{\omega} \operatorname{sech}(\beta r)[(\cos k-1) \sin \Phi+\sin k \cos \Phi] .
$$

Similarly, using (2.6), the current $J_{m, n}$ is given by

$$
J_{m, n} \sim-\frac{2 \epsilon \alpha}{\omega} \operatorname{sech}(\beta r)[(\cos l-1) \sin \Phi+\sin l \cos \Phi] .
$$

The total energy for moving breathers is therefore given by

$$
\begin{aligned}
E^{(0)} \sim & \sum_{m, n} \frac{2 \epsilon^{2} \alpha^{2}}{C_{0}} \cos ^{2} \Phi \operatorname{sech}^{2}(\beta r) \\
& +\frac{2 L \epsilon^{2} \alpha^{2}}{\omega^{2}} \operatorname{sech}^{2}(\beta r)\left[\{(\cos k-1) \sin \Phi+\sin k \cos \Phi\}^{2}\right. \\
& \left.+\{(\cos l-1) \sin \Phi+\sin l \cos \Phi\}^{2}\right]
\end{aligned}
$$

We now approximate the term in square brackets by noting that the average values of $\cos ^{2} \Phi$ and $\sin ^{2} \Phi$ are $\frac{1}{2}$, whilst the average value of $\sin \Phi \cos \Phi$ is zero; the term then simplifies to $\omega^{2} / 2$. Hence, overall, (4.15) becomes

$$
E^{(0)} \sim \sum_{m, n} \frac{2 \epsilon^{2} \alpha^{2}}{C_{0}} \operatorname{sech}^{2}(\beta r)
$$


which is the same expression as the for the energy of a stationary breather (4.10); however, here the definition of $r$, is given by (A.7) so the expression for $E^{(0)}$ is more complex than that used in (4.11). We now replace the double sum over all $(m, n)$ by an integral over $(\xi, \eta)$ and evaluate, (via the substitutions $\epsilon(m-u t)=R \cos \zeta$, $\epsilon(n-v t)=R \sin \zeta)$ to obtain

$$
E^{(0)} \sim \frac{4 \pi \alpha^{2} \log 2}{\beta^{2} C_{0} \omega} \sqrt{\omega^{2} \cos k \cos l-\cos k \sin ^{2} l-\cos l \sin ^{2} k} .
$$

From (A.5) we note that the ratio $\alpha / \beta$ is independent of the envelope parameter $\lambda$; hence, as with stationary breathers, the energy of moving breathers does not depend on the breather amplitude, and so there is a threshold minimum energy required to create a moving breather. The expression (4.17) is strictly positive in the region of ellipticity (see Figure 2.3), has a maximum at $k=l=\pi$ (which corresponds to stationary breathers) and decays to zero at the edge of the domain of ellipticity. Thus the energy threshold for moving breathers is lower than that for stationary breathers. The threshold becomes small as the boundary of the domain of ellipticity is approached.

In section 2 we were only able to find moving breathers for the case of symmetric potential. Thus, assuming $a=0$, we compare (2.28) with (A.1) to find $D=1 / 2 \omega(k, l)$ and $B=3 b \omega(k, l) / 2$. Equation (4.17) thus implies

$$
E^{(0)} \sim \frac{8 \pi(1+2 \log 2) \log 2}{3 b C_{0} \omega^{3}(4 \log 2-1)} \sqrt{\omega^{2} \cos k \cos l-\cos k \sin ^{2} l-\cos l \sin ^{2} k} .
$$

\subsection{Numerical results for symmetric potentials}

Here we present results of numerical simulations of the lattice equations (2.14) for a range of different parameter values, namely symmetric potentials, that is $a=c=0$. Systems with asymmetric potentials $(a, c \neq 0)$ are covered in Section 4.4.

Numerical calculation of conserved quantities is a useful technique for assessing the accuracy of numerical routines. Also, since breathers are spatially localised waveforms, it follows that their energy is also localised. As we shall see, a plot of the cell energy $e_{m, n}$ yields a smooth peak, the centre of which reveals the breather position, which may otherwise be difficult to determine from a plot of the charge $Q_{m, n}$. Plots of cell energy are particularly useful when dealing with moving breathers. To overcome the difficulties outlined above, we choose not to calculate the physically relevant electrical energy given by (4.4). Instead, we note that to leading order, each node displays simple harmonic motion. Hence we define a cell's nondimensional "linear energy" $e_{m, n}^{(\operatorname{lin})}$ as

$$
e_{m, n}^{(\operatorname{lin})}=\frac{1}{2}\left(\frac{\mathrm{d} Q_{m, n}}{\mathrm{~d} t}\right)^{2}+\frac{1}{2} \omega^{2} Q_{m, n}^{2}
$$

where $\omega=\omega(k, l)$ is the expected frequency of the breather as determined by the dispersion relation (2.24). The total energy $E_{l}$ is then the sum of the energies of the unit cells over the entire lattice, that is, $E_{l}=\sum_{m, n} e_{m, n}^{(\operatorname{lin})}$, and this forms a leading 
order approximation of the dimensional energy $E^{(0)}(4.4)$ via $E^{(0)}=E_{l} / C_{0}$. This is the quantity that we calculate in our numerical work. We shall see that determining $e_{m, n}$ and $E_{l}$ helps us in describing the smooth envelope of oscillations and define accurately a position for the localised breather modes.

4.3.1. Stationary breathers in the anomalous dispersive regime. Firstly, we seek stationary breathers in the anomalous dispersion regime - from (3.11), this corresponds to $b>0$. We also impose $k=l=\pi$ so that the velocities $u$ and $v$ are both zero. Following the discussion in Section 3.2, stable solitons are expected when $P K>0$; since $P=5 \epsilon^{2} / 96$, this inequality corresponds to $K>0$, or $51 b^{2}<80 d$; as an example, we set $b=d=1$. The remaining parameters are lattice size $N=30, \epsilon=0.2$ and $\lambda=1$. The variational parameters (A.5) are calculated as $\alpha=1.0517$ and $\beta=3.1399$. The breather frequency is $\omega+\lambda \epsilon^{2}=2.868$, and hence the period of oscillation is $T=2.190$. Figure 4.2(a) shows the initial profile of the breather at the centre of the lattice (as is the case for all our simulations), at which time it is calculated that the breather energy $E_{l}=15.6346$. The breather is also shown after it has completed thirty complete oscillations in Figure 4.2(c). At both times, a plot of the cell energy $e_{m, n}$ is also shown (Figures 4.2(b) and (d)). We observe that the breather is a long-lived mode, which remains highly localised with almost no spreading in any direction, even after long times. Note also that the values obtained for $E_{l}$ do not fluctuate greatly, and even after $30 T, \Delta E_{l}=0.2059$, that is $\Delta E_{l} / E_{l}=0.013$.

4.3.2. Breather moving along a lattice direction. We now show a simulation of a moving breather, which moves along the lattice direction parallel to the $m$-axis (see Figure 4.3). If $\Psi$ denotes the angle made with the direction of travel with the $m$-axis, then it is clear that $\tan \Psi=v / u=\sin (l) / \sin (k)$. We choose wavenumbers $k=\pi / 2$ and $l=\pi$, so that $u=-1 / \sqrt{6} \approx-0.40823, v=0, \Psi=0^{\circ}, \omega=\sqrt{6}$ and $T=\sqrt{2 / 3} \pi$. Also, we set $b=d=1$ (that is, we consider the anomalous dispersion regime, with $P K>0$. The remaining parameters are $N=30, \epsilon=0.1$ and $\lambda=1$. The variational parameters $\alpha$ and $\beta$ are 1.1301 and 2.9220 respectively. The initial profile of the breather is shown in Figure 4.3(a), where the orientation of the internal oscillations can be seen clearly. Note also that the breather envelope is not radially symmetric, rather, it is elongated in the $n$-direction. This is because, in $(k, l)$ parameter space, the breather lies very close to the boundary of the region of ellipticity $\mathcal{D}$ shown in Figure 2.3(c). Clearly, this is a long-lived mode, suffering no appreciable degradation even after sixty seconds or more, and leaving behind very little radiation as it almost completes a circuit of the lattice. Figure 4.3(b) shows the breather as it approaches the left hand edge of the lattice. It is not possible to read the exact position of the breather from a plot of $Q_{m, n}(t)$ with great precision. Nevertheless, one may observe that by this time, the breather has traversed approximately ten sites. The theoretical value for the velocity is calculated as -0.40823 units per second, by which time the breather should have travelled 9.5 units, which is in good agreement with the measured value. Figure 4.3(c) shows the breather disappearing 


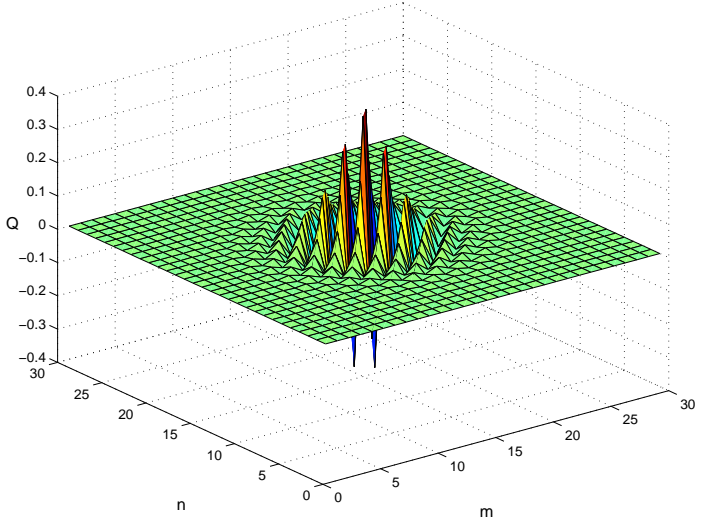

(a) Profile at $t=0$

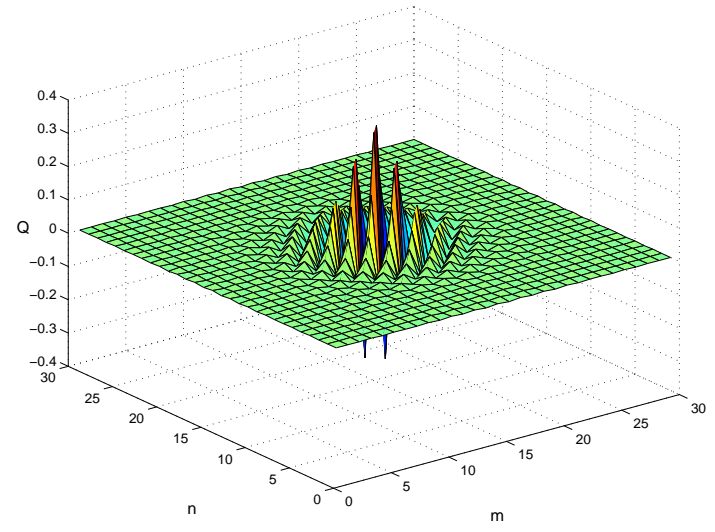

(c) Profile at $30 T=65.714$

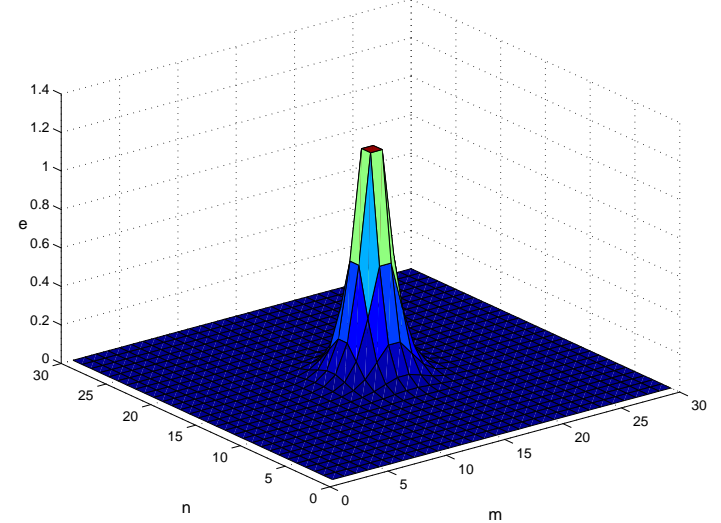

(b) Plot of $e_{m, n}, E_{l}=15.6346$

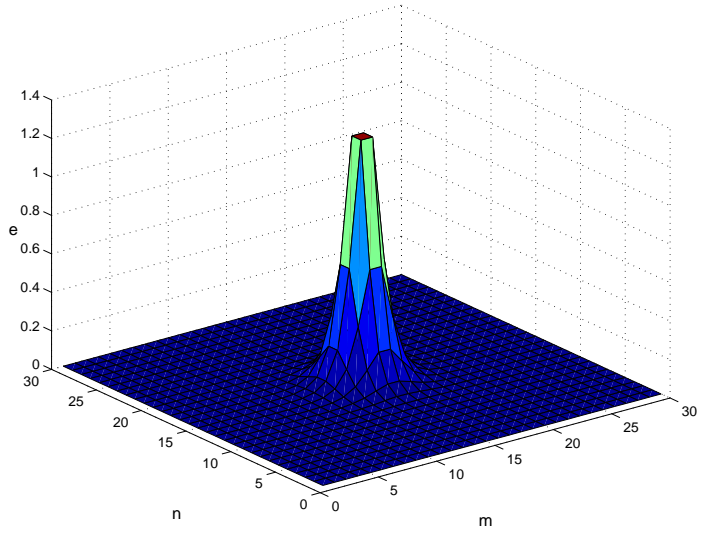

(d) Plot of $e_{m, n}, E_{l}=15.8405$

Figure 4.2. Stationary breather in the anomalous dispersive regime.

from the left hand edge and reappearing from the right, owing to the periodic boundary conditions. After approximately sixty seconds, the breather has completed nearly a full circuit, remaining localised with almost no spreading, only leaving a little radiation in its wake, (see Figure 4.3(d)). It is not clear from these simulations whether the shedding of radiation is due to a short-term transient effect as our approximate initial conditions adjust to the true shape of a travelling breather, or whether the breather will continue to lose energy as it moves through the lattice.

4.3.3. Breathers moving at $\Psi=45^{\circ}$ to the lattice. As mentioned after (2.25), the angle of propagation of the envelope $\Psi$ is given by $\tan ^{-1}(\sin (l) / \sin (k))$. In order to investigate the direction $\Psi=45^{\circ}$, we choose $k=l$. Bearing the results of the previous simulation in mind, we choose a point in $(k, l)$-parameter space not too near the boundary of ellipticity, otherwise the breather envelope will be elongated in one direction. Hence, 


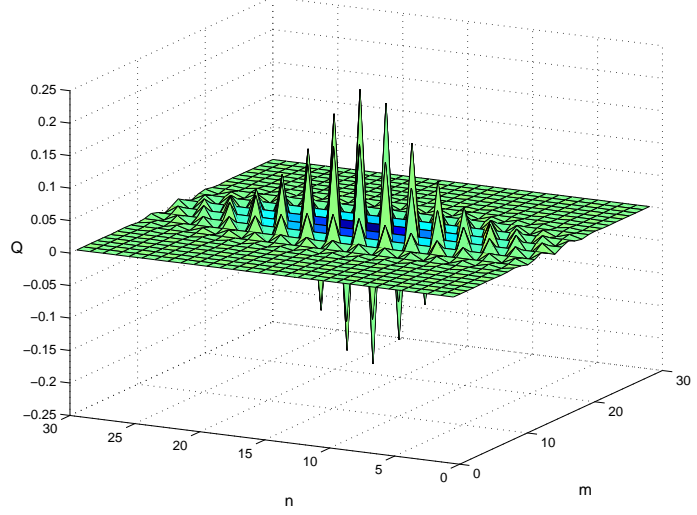

(a) Initial profile, $t=0$

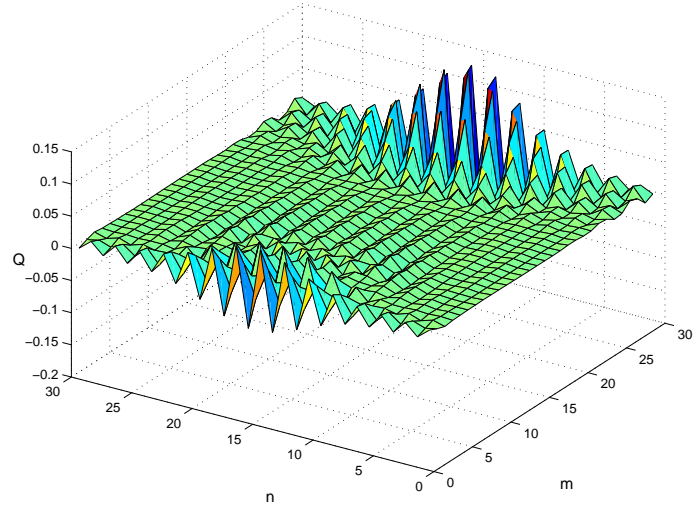

(c) Profile at $t=17.04 T=43.7172$

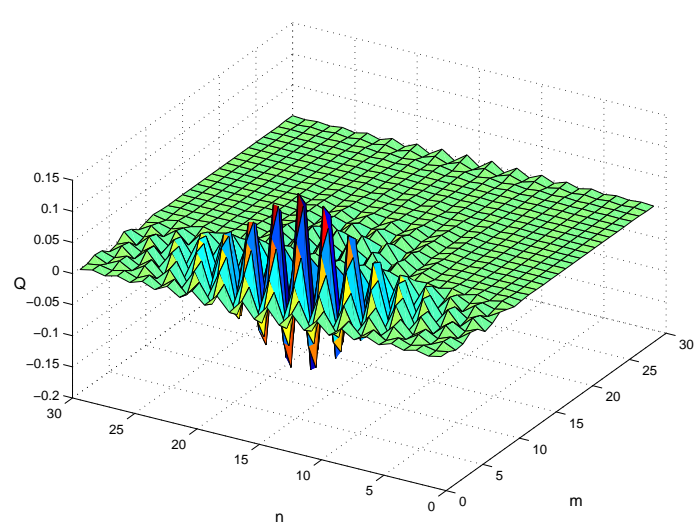

(b) Profile at $t=9.084 T=23.3030$

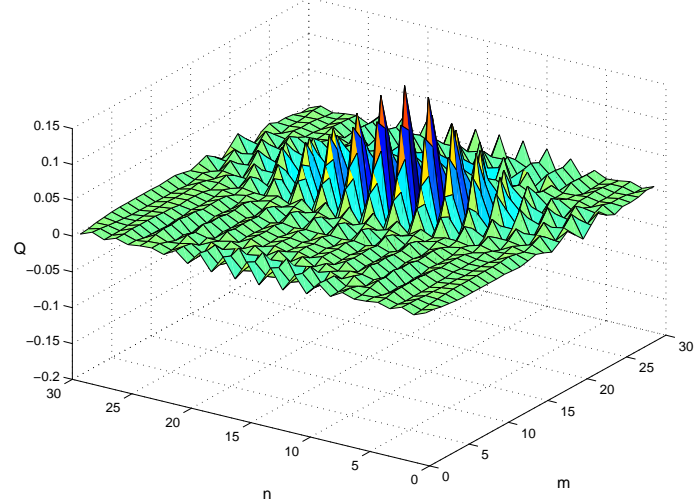

(d) Profile at $t=24.70 T=63.3464$

Figure 4.3. Breather moving along a lattice direction, $\Psi=0^{\circ}$.

we select $k=l=3 \pi / 4$, and for the same reasons as before, we set $b=d=1, N=30$, $\epsilon=0.1$ and $\lambda=1$. The variational parameters are $\alpha=1.0941$ and $\beta=3.0180$, and the horizontal and vertical velocities are both -0.2706 units per second. The breather starts at the centre of the lattice (not shown), and is shown at times 40, 60 and 80 seconds in Figure 4.4. It can be seen that the breather remains a localised coherent structure even after 80 seconds, without any obvious degradation as it completes a whole loop.

We wish to track the position of the breather at each stage of its motion. A plot of the cell energy $e_{m, n}$ against $m, n$ allows the position of the breather to be determined. As an example, a plot of $e_{m, n}$ at $t=20$ is given in Figure 4.4(e). In practise, greater accuracy can be achieved through the use a plot of $e_{m, n}^{3}$ since this is more sharply peaked than $e_{m, n}$ (see Figure 4.4(f)). The horizontal and vertical displacement of the breather 


\begin{tabular}{|c|c|c|c|c|}
\hline $\begin{array}{c}\text { Time } \\
(\mathrm{s})\end{array}$ & $\begin{array}{c}\text { horizontal } \\
\text { displacement }\end{array}$ & $\begin{array}{c}\text { vertical } \\
\text { displacement }\end{array}$ & $\begin{array}{c}\text { av. horiz. } \\
\text { velocity }\end{array}$ & $\begin{array}{c}\text { av. vert. } \\
\text { velocity }\end{array}$ \\
\hline $20 \mathrm{~s}$ & -4 units & -4 units & -0.2 units s $^{-1}$ & -0.2 units s$^{-1}$ \\
\hline $40 \mathrm{~s}$ & -9 units & -9 units & -0.225 units s $^{-1}$ & -0.225 units s $^{-1}$ \\
\hline $60 \mathrm{~s}$ & -16 units & -16 units & -0.267 units s $^{-1}$ & -0.267 units s$^{-1}$ \\
\hline $80 \mathrm{~s}$ & -21 units & -21 units & -0.2625 units s $^{-1}$ & -0.2625 units s$^{-1}$ \\
\hline
\end{tabular}

Table 1. Summary of breather motion $\left(\Psi=45^{\circ}\right)$

is recorded every twenty seconds, and the average speed is computed along with the direction of motion. Table 1 summarises the motion of the breather. Clearly, $\tan \Psi=1$ and hence $\Psi=45^{\circ}$. The final computed value for the velocities is -0.2625 units per second, which is very close to the theoretical prediction of -0.2706 units per second (a relative error of $3 \%$ ).

4.3.4. Breather moving at $\Psi=20^{\circ}$ to the lattice. Following the work of Marin et al. [24], a natural question to consider is whether the lattice supports breathers moving in directions which are not axes of symmetry of the lattice. Since the angle of propagation of the envelope $\Psi$ is $\tan ^{-1}(\sin (l) / \sin (k))$, it is easily verified that within the region $\mathcal{D}$ shown in Figure 2.3(c), any angle $\Psi \in[0,2 \pi]$ is possible. In other words, from the asymptotic analysis of Section 2.3 it appears that there is no restriction upon the direction in which breathers may travel in the lattice. Our numerical work supports this hypothesis. This is in stark contrast to the scenario reported for the two-component lattice by Marin et al. [24].

We have tried propagating breathers in a range of directions, including at an angle $\Psi=20^{\circ}$ to the $m$-axis, shown in Figure 4.5. The numerical scheme was initiated with $k=3 \pi / 4$ and $l=2.881$ radians. It may be checked that for this choice of wavenumbers, the theoretical values for $u$ and $v$ are -0.2609 and -0.0950 respectively, and the calculated angle of travel is indeed $20^{\circ}$. Also, this point in $(k, l)$-space lies well within the region of ellipticity; the linear carrier wave has a frequency $\omega=2.710$ and so the time period for an oscillation is $T=2.318$. Once more, we set $b=d=1$, $N=30, \epsilon=0.1$ and $\lambda=1$, and the breather is initially located at the centre of the lattice. The variational parameters are $\alpha=1.0743$ and $\beta=3.0737$. Figure 4.5 shows the breather at times $t=40,75,120$ and 160 seconds. Clearly the breather moves through the lattice and preserves its form remarkably well, leaving behind very little radiation as it moves, nor spreading noticeably in any direction. The motion of the breather is summarised in Table 2 . The final measurement for the average velocities $u$ and $v$ are -0.2594 and -0.0969 units per second respectively, yielding relative errors of $1 \%$ and $2 \%$. The direction of travel is measured to lie at $20.479^{\circ}$ to the lattice. These results suggest that breathers can propagate at any direction in the lattice. 


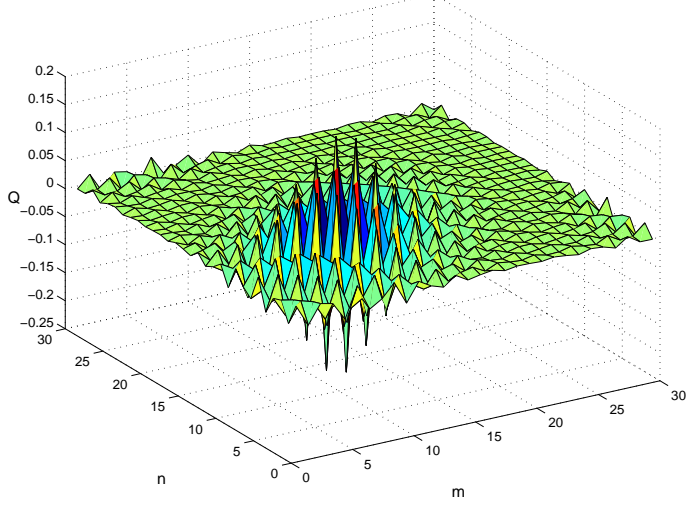

(a) Profile at $t=8.318 T=20$

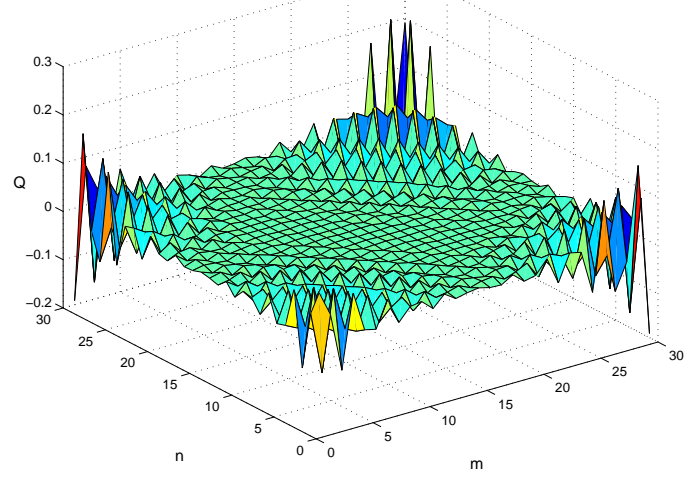

(c) Profile at $t=24.95 T=60$

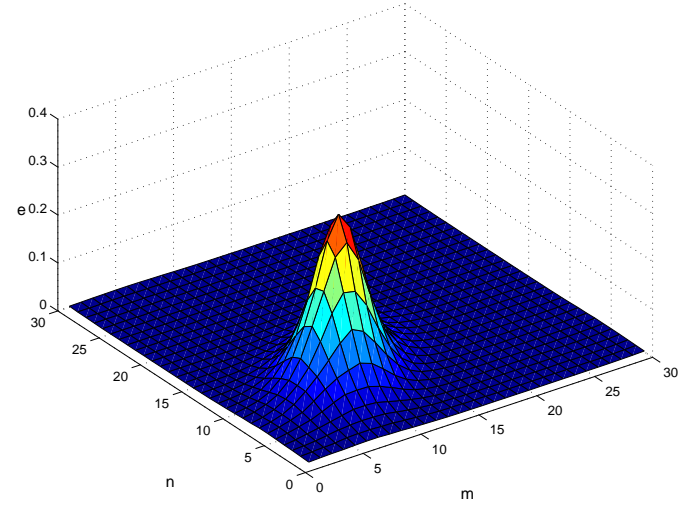

(e) Plot of $e_{m, n}$ at $t=8.318 T=20$

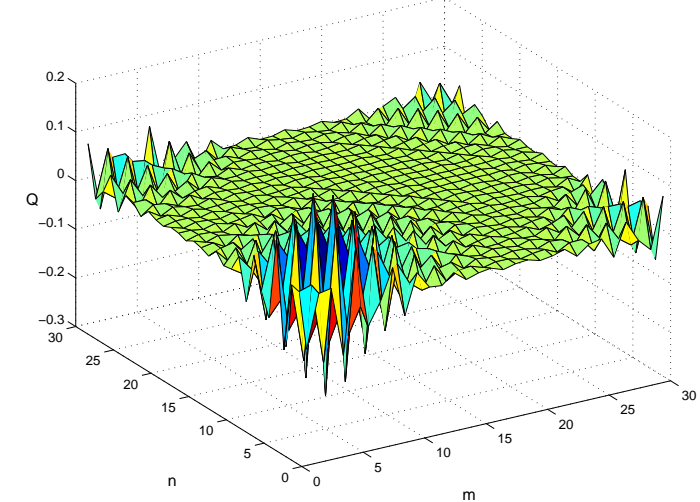

(b) Profile at $t=16.64 T=40$

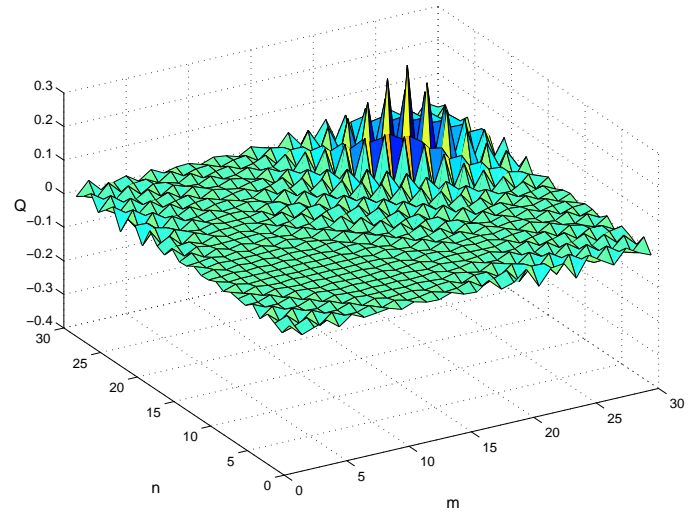

(d) Profile at $t=33.27 T=80$

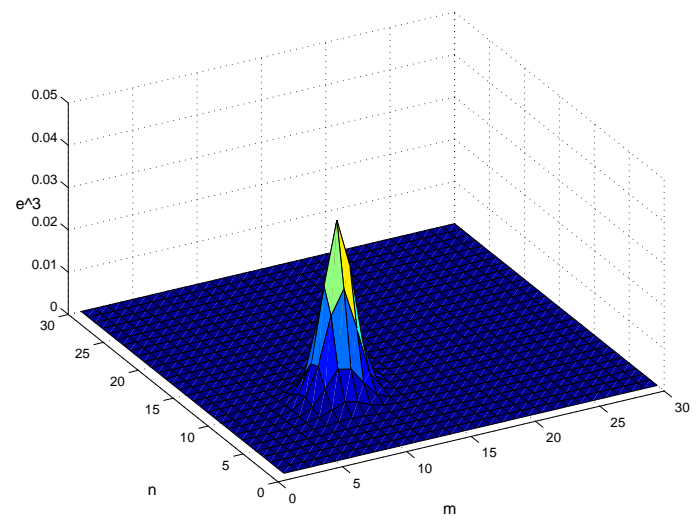

(f) Plot of $e_{m, n}^{3}$ at $t=8.318 T=20$

Figure 4.4. Breather moving at $\Psi=45^{\circ}$ to the lattice: $t=40,60$ and 80 seconds. 


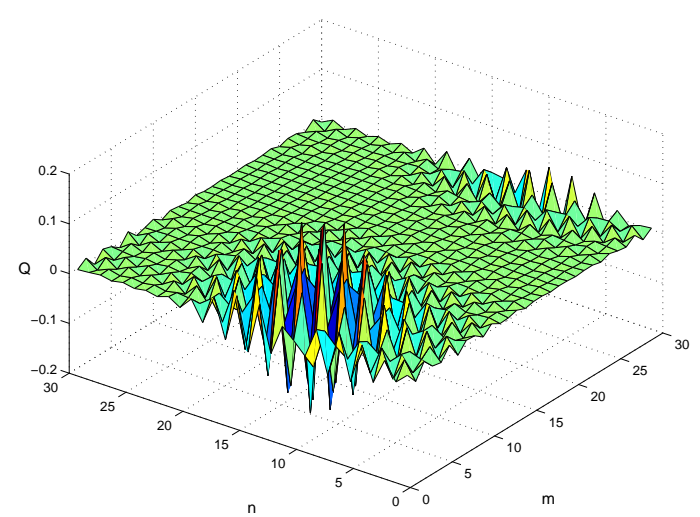

(a) Profile at $t=17.26 T=40$

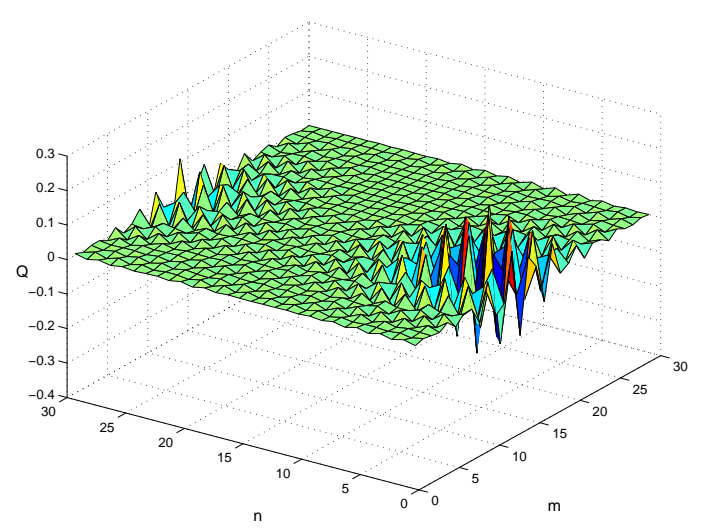

(c) Profile at $t=51.77 T=120$

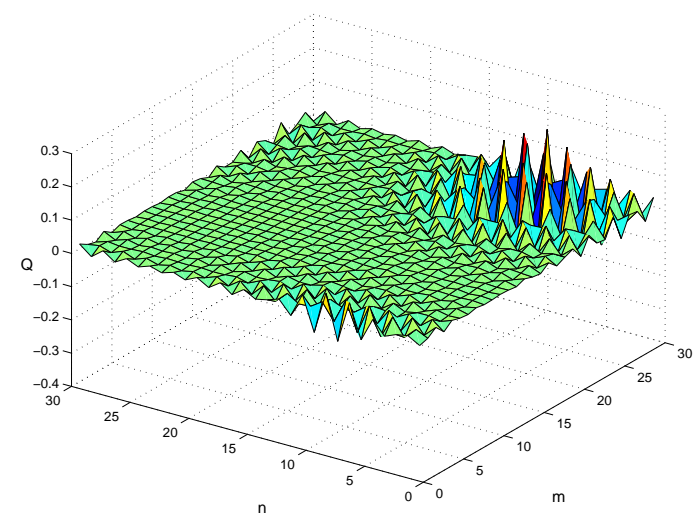

(b) Profile at $t=32.35 T=75$

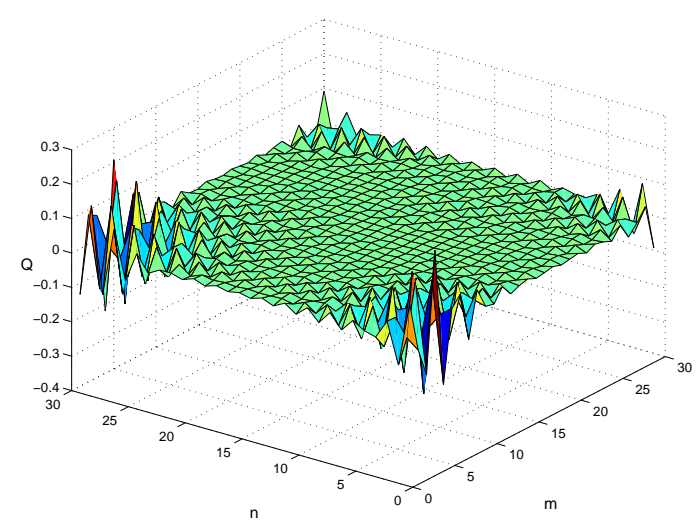

(d) Profile at $t=69.02 T=160$

Figure 4.5. Breather moving at $\Psi=20^{\circ}$ to the lattice.

\begin{tabular}{|c|c|c|c|c|c|c|}
\hline $\begin{array}{c}\text { Time } \\
(\mathrm{s})\end{array}$ & $\begin{array}{c}\text { horiz. } \\
\text { disp. }\end{array}$ & $\begin{array}{c}\text { vert. } \\
\text { disp. }\end{array}$ & $\begin{array}{c}\text { av. horiz. } \\
\text { vel. (units/s) }\end{array}$ & $\begin{array}{c}\text { av. vert. } \\
\text { vel. (units/s) }\end{array}$ & $\tan \Psi$ & $\Psi$ \\
\hline $40 \mathrm{~s}$ & -10 & -3 & -0.25 & -0.075 & 0.3 & $16.70^{\circ}$ \\
\hline $75 \mathrm{~s}$ & -19 & -6.5 & -0.2533 & -0.0867 & 0.3421 & $18.89^{\circ}$ \\
\hline $120 \mathrm{~s}$ & -31 & -11 & -0.2583 & -0.09167 & 0.3548 & $19.54^{\circ}$ \\
\hline $160 \mathrm{~s}$ & -41.5 & -15.5 & -0.2594 & -0.0969 & 0.3735 & $20.48^{\circ}$ \\
\hline
\end{tabular}

Table 2. Summary of breather motion $\left(\Psi=20^{\circ}\right)$ 


\subsection{Numerical results for asymmetric potentials}

The above numerical results all feature symmetric potentials, namely, those for which $a=c=0$ so that $V(-Q)=-V(Q)(2.12)$. We now present results of a simulation for which the potential function is asymmetric. Recall from Section 2.6 that the lattice equations (2.14) can be reduced to a cubic two-dimensional NLS equation in $F(2.33)$ for asymmetric potentials for the special case $k=l=\pi$ which corresponds to the case of stationary breathers $(u=v=0)$. The breather frequency $\omega$ and period $T$ are 2.868 and 2.190 respectively. Equation (2.33) has the same form as (3.1) with $D=\sqrt{2} / 8$ and $B=\sqrt{2}\left(3 b-4 a^{2}\right)$. Applying the same method as outlined in Appendix A, soliton solutions of (2.33) have the form (A.6), where $D=\sqrt{2} / 8, B=\sqrt{2}\left(3 b-4 a^{2}\right)$, and $r=\sqrt{\xi^{2}+\eta^{2}}=\sqrt{X^{2}+Y^{2}}$. Also from Section 2.6, $G_{0}=-a|F|^{2}$ and $G_{2}=0$, and so $Q_{m, n}$ can be found to second order,

$$
Q_{m, n}(t)=2 \epsilon \alpha \cos \left[\pi m+\pi n+\left(\omega+\epsilon^{2} \lambda t\right)\right] \operatorname{sech}(\beta r)-2 a \epsilon^{2} \alpha^{2} \operatorname{sech}^{2}(\beta r) \cdot(4
$$

Surprisingly the presence of the quadratic nonlinearity $(a \neq 0)$ does not generate any second harmonic term $\left(G_{2}=0\right)$, but does cause a small shift of the oscillation to lower $Q$ values.

The anomalous dispersive regime corresponds to the region in $(a, b)$-parameter space where $3 b>4 a^{2}$. We set $a=1, b=2, c=0$ and $d=1$. The remaining parameters are assigned the values $N=40, \epsilon=0.1$ and $\lambda=1$, and the variational parameters are $\alpha=1.2880$ and $\beta=3.1399$. The breather is initially situated at the centre of the lattice (not shown), at which time the energy $E_{l}$ is 23.6712. The breather is shown after it has completed ten, thirty and forty complete oscillations (see Figure 4.6). Again, it can be seen from the corresponding plots of $e_{m, n}$ that the breather remains localised, with very little loss of form, even after almost ninety seconds. As with static breathers in the lattice with a symmetric potential, very little energy is shed in the form of radiation. Note that the breather energy $E_{l}$ remains more or less unchanged, and after forty oscillations, $\Delta E_{l} / E_{l}=0.01$.

\subsection{Breather collisions}

One can gain insight as to breather stability and robustness by simulating collisions between two (or more) breather modes. In particular, we propose to investigate the robustness of breathers by simulating the collision of two breathers. Interesting questions include: whether the breathers retain their form following a collision, whether the velocities are altered significantly, whether stability depends upon the relative orientation of incoming breather paths, etc.

The first collision is between a breather is initially located towards the left-hand edge of the lattice, at the grid point $(7.5,15)$. and a breather near the right-hand edge of the lattice, at the point $(22.5,15)$. We observe what happens when the two breathers move towards one another parallel to the $m$-axis, and interact. For this reason, we choose $k_{L}=5 \pi / 4, l_{L}=\pi$, and $k_{R}=3 \pi / 4, l_{R}=\pi$; hence $\omega=2.7229$ and $T=2.3075$. 


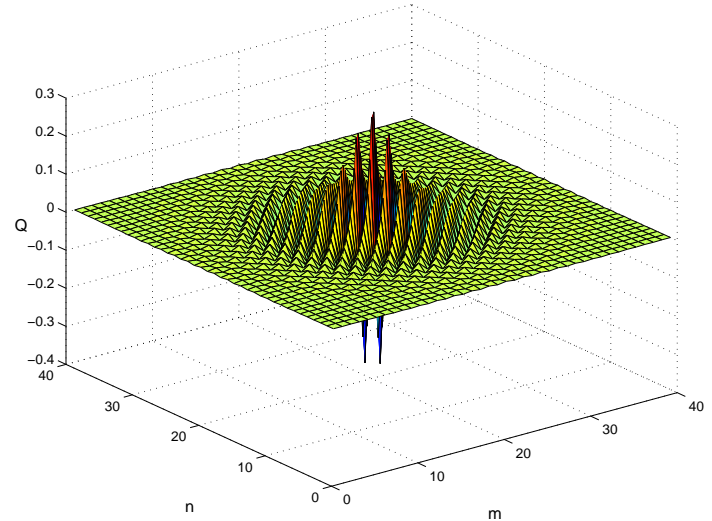

(a) Profile at $t=10 T=21.905$

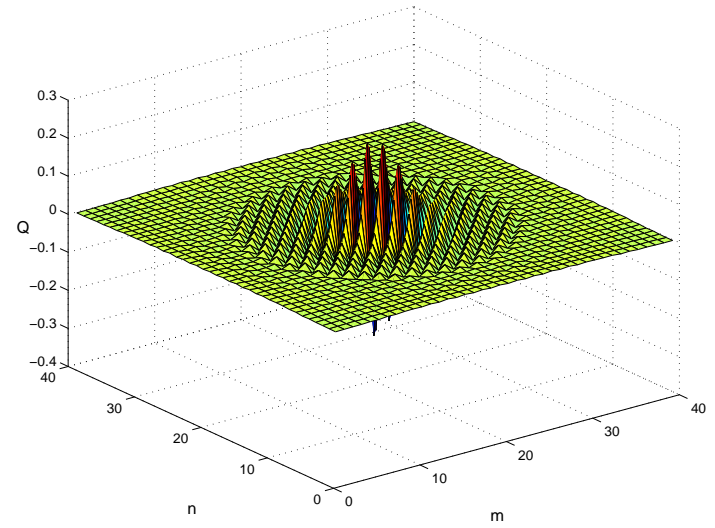

(c) Profile at $t=30 T=65.714$

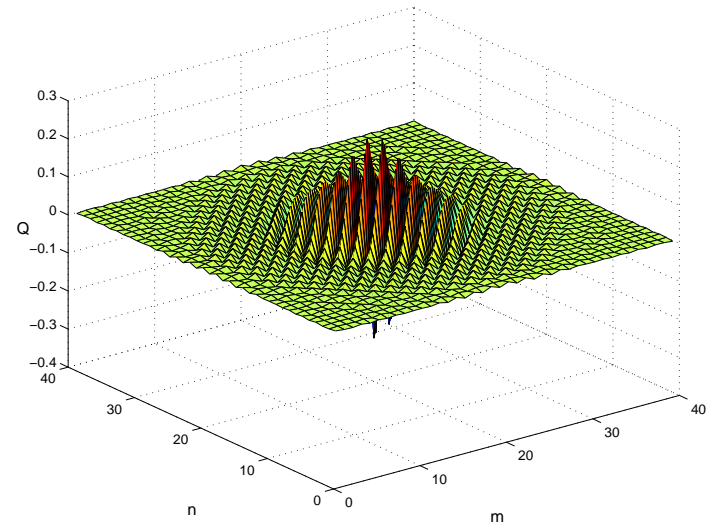

(e) Profile at $t=40 T=87.619$

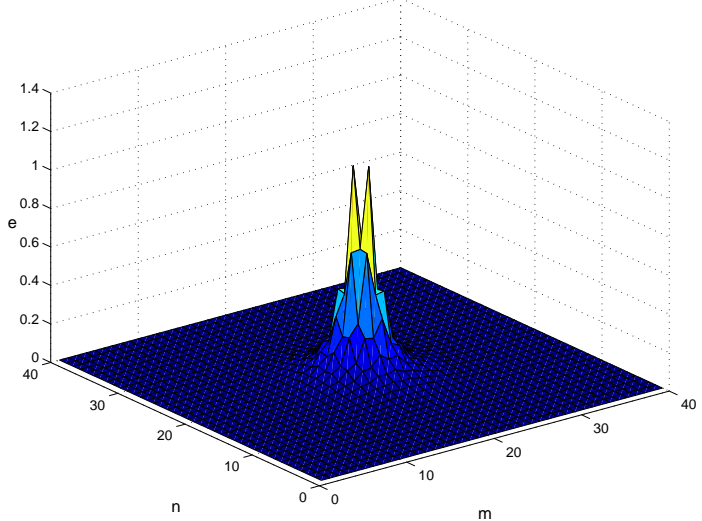

(b) Plot of $e_{m, n}, E=23.4796$

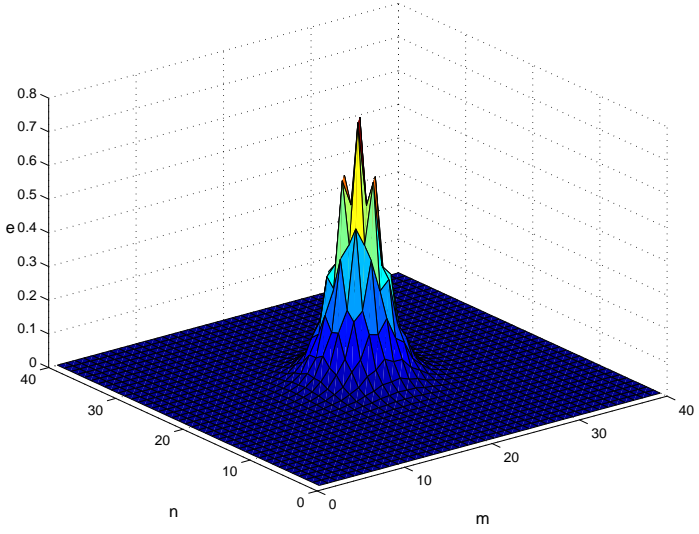

(d) Plot of $e_{m, n}, E=23.9433$

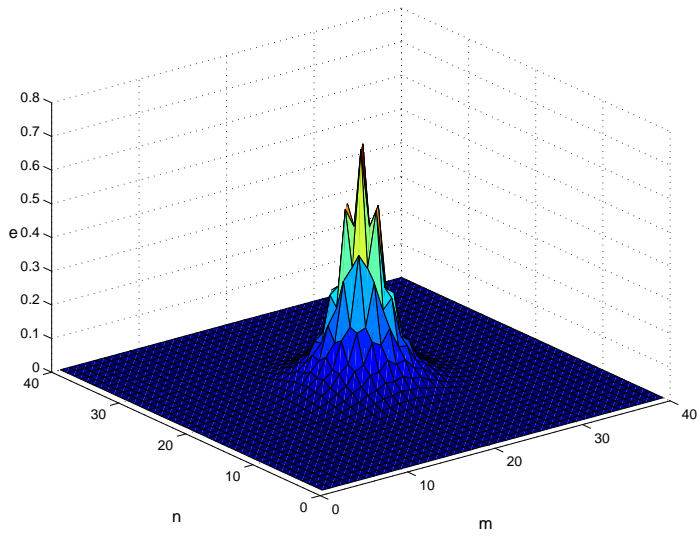

(f) Plot of $e_{m, n}, E=23.8096$

Figure 4.6. Stationary breather in the anomalous dispersive regime for an asymmetric potential. 


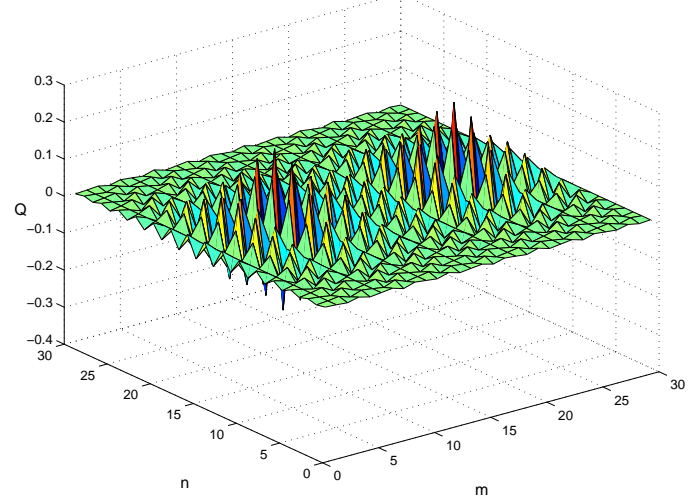

(a) Profile at $t=0$

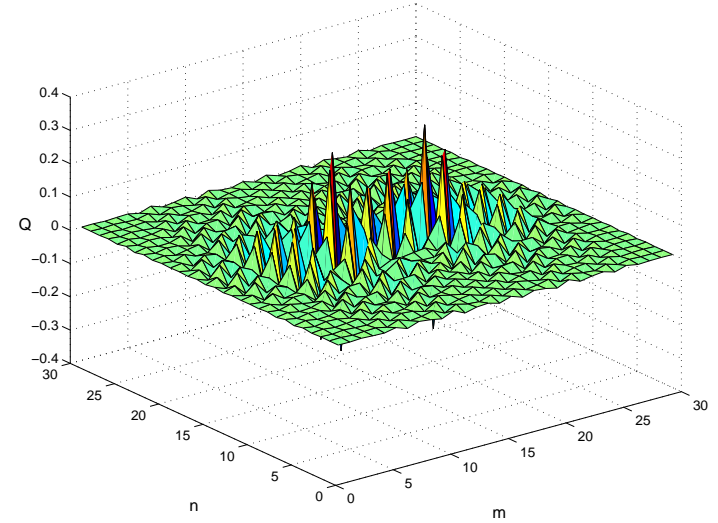

(c) Profile at $t=19.2 T=44.3041$

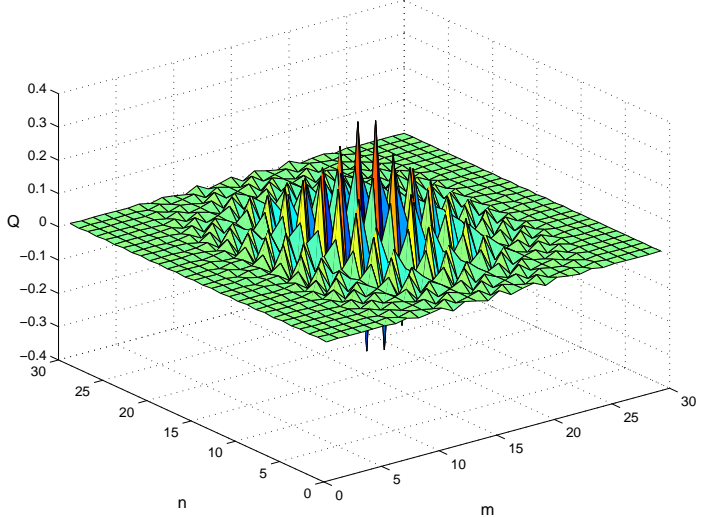

(b) Profile at $t=11.48 \mathrm{~T}=26.489$

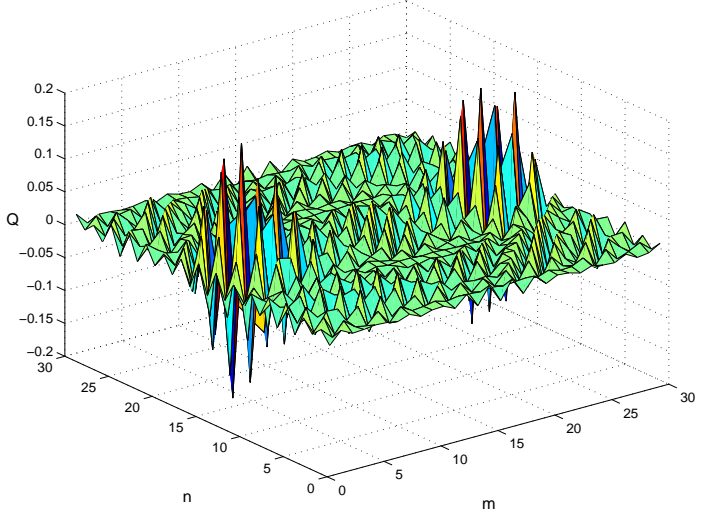

(d) Profile at $t=30.62 \mathrm{~T}=70.6554$

Figure 4.7. Colliding breathers interact and separate.

The corresponding velocities of the $\mathrm{LH}$ and $\mathrm{RH}$ breather are 0.2597 and -0.2597 units per second respectively. The initial configuration of the lattice is shown in Figure 4.7(a). The breathers approach and undergo a complicated interaction as they collide, as shown at various intervals in Figure 4.7. They then pass through one another and begin to separate, as seen clearly in Figure 4.7(d). Here, they can be seen as two visibly distinct structures in the lattice, after having emerged with very little overall change. Clearly, energy is shed during the collision, but this is to be expected since the FPU system in general is known not to be integrable. Measurements reveal that the velocities of both breathers are preserved even after collision. The picture becomes complicated for longer times because of the periodic boundary conditions meaning that the breathers emerging from the first collision will collide again at the boundary. 


\section{Discussion}

In this paper our aim has been to find criteria for the existence of breathers in the two-dimensional Fermi-Pasta-Ulam equation (2.14). In carrying out a small amplitude asymptotic expansion, we have found various conditions on the lattice interaction parameters $a, b, c, d$, and on the form of the carrier wavenumbers $k$ and $l$. We have also found a particular modified NLS equation (3.11) which requires more detailed analysis. The results have been supplemented and supported by numerical simulations.

Equation (2.14) comprises a system of infinitely many coupled second-order nonlinear ordinary differential equations. Exact breather solutions of this system cannot be found explicitly. If an analytic approach is to be employed, a continuum approximation must be applied at some stage, otherwise the system of equations is not amenable to analysis. In one-dimensional systems, few difficulties arise, since the resulting continuum PDE, the one-dimensional NLS equation is essentially well-behaved, being integrable and having explicit soliton solutions (Butt \& Wattis [30]).

In Sections 2.4 and 2.6, we have showed that by applying the method of multiplescales in conjunction with a continuum approximation the two-dimensional Fermi-PastaUlam equation (2.14) can be reduced to a cubic NLS equation at third-order. As explained in Section 3.2, this continuum partial differential equation exhibits blow up and dispersion. We have shown that these problems may be overcome by including higher-order effects. Our motivation comes from a review of results available for generalised NLS equations which incorporate higher-order nonlinearity and dispersion.

The NLS equation (3.1) is generic in that it describes the envelope of a wavepacket in a model which takes into account nonlinearity and dispersion at the lowest orders. It thus arises in a whole variety of contexts, ranging from deep-water gravity waves (Yuen \& Lake [45]), to nonlinear optics (Chiao et al. [31]), to plasma physics, Zakharov [46]. However, the derivation of higher order equations yields many possible perturbations, not all of which have been considered in the literature. Generality of the cubic two-dimensional NLS equation is lost when higher-order terms are included. For some perturbations it is known that blow-up is suppressed, and stable solitons exist. Unfortunately the higher-order equation we derive (3.11) has several perturbing terms, and as far as we are aware, only some of the perturbing terms have been previously studied in the literature. Our equation (3.11) is derived by applying a fifth-order multiple-scale analysis to the two-dimensional Fermi-Pasta-Ulam equations (2.14). The analysis of (3.11), namely the rigorous determination of which parameter values (if any) support stable soliton solutions is left for future work. We mentioned in Section 3.3 that the modulation theory described by Fibich and Papanicolaou $[44,35]$ might be suitable for analysis of (3.11). On the other hand, if a variational formulation can be found for (3.11), then the methods of Kuznetsov et al. [42] could be applied. Of course, a numerical analysis of the modified NLS equation is another possible approach, but then one may as well bypass analysis of the continuum equation (3.11), and instead numerically solve the lattice equations (2.14) to begin with. 
There are other possibilities which may be pursued in addition. We mention the work of Tamga et al. [10], who apply a simple one-term multiple-scale expansion to a two-dimensional lattice. They show that the lattice equations can be reduced to a cubic two-dimensional NLS equation at third order, which they recognise "cannot be used to predict the time evolution" of nonlinear localised modes. They instead consider modulational instability of their NLS equation, and (aided by numerical simulations), show that localised modes exist in the regions where a uniform solution is unstable. As pointed out by Marin et al. [25], the drawback to this approach is that the "nonlinear localised modes" identified cannot reliably be identified as discrete breathers. The authors of [25] thus describe the modes as "breathing solitary waves," rather than discrete breathers.

Several interesting breather properties are presented in the numerical results of Section 4. Firstly, we note that stationary and moving breathers appear to be longlived modes of the lattice; their profiles do not change over time and they shed very little energy as they evolve. We have shown this to be true for a range of different parameter values, including asymmetric as well as symmetric potentials. The fact that breather modes are observed to reform following collisions supports the claim that these modes are robust structures. One of the more surprising results that emerges from the numerics is that there appears to be no restriction upon the direction in which breathers can travel in the lattice, in contrast to the scenario reported by Marin [24, 25] for two-component two-dimensional lattices. We have found, for instance, breathers which move at $20^{\circ}$ to the lattice, suffering no appreciable distortion or spreading, even after relatively long times. In fact, we have obtained breathers moving at many other angles not parallel to the lattice axes, though we have not presented results of those simulations here. Breathers which move along the lattice direction are elongated in the direction perpendicular to their motion. Previous work on the two-dimensional lattice analysed here has focused on other types of excitation. In [47] Eilbeck found highly accurate numerical approximations to the shape of travelling solitary plane waves. These were also found to travel at any angle to the lattice, and showed a more subtle angle-dependence in their amplitude-speed characteristics which was explained using high-order quasi-continuum expansions [48].

In Section 4 we have also derived an asymptotic expression for the energy of stationary and moving breathers. This has the expected form that the energy does not vanish as the amplitude becomes arbitrarily small. Rather, there is a threshold energy required to create a breather, in agreement with the results of Flach et al [21]. We have provided formulae for these energy thresholds (sections 4.2.1 and 4.2.2), and contrary to what one might expect, the energy threshold for moving breathers is smaller than that for stationary breathers. It is independent of the breather amplitude, but is dependent on the wavenumbers, and becomes vanishingly small as one approaches the boundary of the domain of ellipticity.

Detailed analyses of the mobility of breathers have been undertaken by a number of authors, see $[49,50,51]$ for examples of such studies of one-dimensional breathers. 
Aubry and Cretegny analyse the stability of a breather using Floquet multipliers and find highly mobile breathers at the instability thresholds of the immobile breathers [50]. Flach and Willis [49] consider the separatrix between immobile and mobile breathers using a simple Hamiltonian system of the three coupled oscillators at the centre of a breather. From the form of this separatrix, they deduce that no simple Peierls-Nabarro $(\mathrm{PN})$ potential can be constructed. Flach and Gorbach [51] analyse the stability of the Page mode (a breather whose centre lies between two lattice sites) and the SeiversTakeno mode (a breather whose centre lies at a lattice site). They find that if the FPU potential has no cubic term then the Page model is stable and the Sievers-Takeno mode is unstable, the type of situation one might expect from an approach based on Peierls-Nabarro (PN) energetics. However, if the FPU potential has a non-trivial cubic component, Flach and Gorbach find that both modes are unstable. This result suggests that defining PN energies for slowly-moving breathers is less useful than for travelling waves. Where PN energies have been calculated for moving breathers (such as in [52]), their complicated form indeed makes interpretation difficult, confirming the speculation of Flach and Willis in [49]. We argue that any analysis of breather mobility in terms of PN energies would require the kinetic energy as well as potential energy to included (as carried out in [52]) and further, if possible, the kinetic energy to be separated into two components - one due to internal oscillations of the breather and a component for the bulk motion of the breathers position. The concept of Peierls-Nabarro energies for moving breathers is more difficult in higher-dimensions than for one-dimensional systems; since, depending on the direction of motion through the lattice, a breather may not be periodic at all, or may be periodic with a very long period.

\section{Acknowledgments}

IAB would like to acknowledge financial assistance from the UK Engineering and Physical Sciences Research Council. We would like to thank the referees for their perceptive comments and pointing out some useful extra references.

\section{Appendix A. Approximate analytic formulae for Townes solitons}

Unfortunately, analytic formulae are not known for the family of Townes soliton solutions of (3.1), and therefore some other method must be used to generate an approximate analytic solution. We use the Rayleigh-Ritz variational method to do this. Suppose we look for time-harmonic radially symmetric solutions of (3.1) of the form $F(\mathbf{x}, T)=e^{i \lambda T} \phi(r)$, where $r=|\mathbf{x}|=\sqrt{\xi^{2}+\eta^{2}}$. Substituting for $F$ in (3.1) yields

$$
-\lambda \phi+D \nabla^{2} \phi+B \phi^{3}=0
$$

where $\nabla^{2}=\partial^{2} / \partial \xi^{2}+\partial^{2} / \partial \eta^{2}$. Equation (A.1) has a variational structure, and the corresponding action integral $\mathcal{E}$ has the form

$$
\mathcal{E}(\phi)=\int \frac{1}{2} \lambda|\phi|^{2}+\frac{1}{2} D|\nabla \phi|^{2}-\frac{1}{4} B|\phi|^{4} d^{2} \mathbf{r}
$$


We use a radially symmetric trial solution of the form $\phi=\alpha \operatorname{sech}(\beta r)$, where $\alpha$ and $\beta$ are parameters to be determined and $r=\sqrt{\xi^{2}+\eta^{2}}$. Substituting this form for $\phi$ into (A.2), and evaluating the integral (using Maple to evaluate the resulting integrals) gives an expression for $\mathcal{E}$ in terms of the variational parameters $\alpha$ and $\beta$. It can be shown that

$$
\mathcal{E}(\alpha, \beta)=\frac{D(1+2 \ln 2)}{12} \alpha^{2}-\frac{Q(4 \ln 2-1)}{24} \frac{\alpha^{4}}{\beta^{2}}+\frac{\lambda \ln 2}{2} \frac{\alpha^{2}}{\beta^{2}}
$$

The soliton solution corresponds to a stationary point of the action $\mathcal{E}$, and therefore the parameters $\alpha$ and $\beta$ are determined by the equations

$$
\frac{\partial \mathcal{E}}{\partial \alpha}=\frac{\partial \mathcal{E}}{\partial \beta}=0
$$

Differentiating $\mathcal{E}$ with respect to $\beta$ results in an equation for $\alpha^{2}$ from which $\alpha$ is found, and once $\alpha$ is known, $\beta$ can be determined from the equation $\partial \mathcal{E} / \partial \alpha=0$, yielding

$$
\alpha=\sqrt{\frac{12 \lambda \ln 2}{B(4 \ln 2-1)}} \text { and } \beta=\sqrt{\frac{6 \lambda \ln 2}{D(2 \ln 2+1)}} .
$$

Hence an approximate form for the Townes soliton solution of (3.1) is

$$
F=\sqrt{\frac{12 \lambda \log 2}{B(4 \log 2-1)}} \exp (i \lambda T) \operatorname{sech}\left(\sqrt{\frac{6 \lambda \log 2}{D(2 \log 2+1)}} \sqrt{\xi^{2}+\eta^{2}}\right) .
$$

As an example, let us consider the lattice with a symmetric potential, whose envelope is governed by (2.26). On seeking a solution of the form $F=\mathrm{e}^{i \lambda T} \phi(r)$ with $r^{2}=\xi^{2}+\eta^{2}$ we find

$$
r^{2}=\xi^{2}+\eta^{2}=\frac{\left[v^{2}-\cos l\right] Z^{2}+\left[u^{2}-\cos k\right] W^{2}-2 u v Z W}{\cos k \cos l-u^{2} \cos l-v^{2} \cos k} .
$$

Substituting the soliton solution for $F$ (A.6) into the lattice ansatz (2.18) gives the leading order analytic expression as

$$
Q_{m, n}(t)=2 \epsilon \alpha \cos \left[k m+\ln +\left(\omega+\epsilon^{2} \lambda\right) t\right] \operatorname{sech}(\beta r),
$$

where $\alpha$ and $\beta$ are given in (A.5), $Z=\epsilon(m-u t), W=\epsilon(n-v t), u=-\sin k / \omega$, $v=-\sin l / \omega$ and $\omega^{2}=4 \sin ^{2}(k / 2)+4 \sin ^{2}(l / 2)$.

For the lattice with asymmetric potential, since only the case $k=l=\pi$ is considered and $u=v=0$, the transformation (2.27) reduces to the trivial $\xi=Z=X, \eta=W=Y$.

\section{References}

[1] E Fermi, J Pasta, J \& S Ulam. 1955. Studies in nonlinear problems. Los Alamos Scientific Laboratory report LA-1940. Reprinted in Lectures in Applied Mathematics, 1974, 15, 143.

[2] S Takeno, K Kisoda \& AJ Sievers. 1988. Intrinsic localized vibrational modes in anharmonic crystals. Prog Theor Phys Suppl, 94, 242.

[3] S Takeno \& K Hori. 1991. Self-localized modes in a pure one-dimensional lattice with cubic and quartic lattice anharmonicity. J Phys Soc Japan, 60, 947.

[4] AA Ovchinnikov. 1970. Localized long-lived vibrational states in molecular crystals. Sov Phys JETP, 30, 147. 
[5] S Flach \& CR Willis. 1998. Discrete breathers. Phys Rep, 295, 181.

[6] S Takeno. 1990. Localized modes in the long-time behaviour of anharmonic lattices. J Phys Soc Japan, 59, 1571.

[7] S Takeno. 1992. Theory of stationary anharmonic localized modes in solids. J Phys Soc Japan, 61, 2821.

[8] VM Burlakov, SA Kiselev \& VN Pyrkov. 1990. Computer simulation of intrinsic localized modes in one-dimensional and two-dimensional anharmonic lattices. Phys Rev B, 42, 4921.

[9] D Bonart, AP Mayer \& U Schröder. 1995. Anharmonic localized surface vibrations in a scalar model. Phys Rev B, 51, 13739.

[10] J M Tamga, M Remoissenet \& J Pouget. 1994. Breathing solitary waves in a sine-Gordon twodimensional lattice. Phys Rev Lett, 75, 357.

[11] AA Ovchinnikov \& S Flach. 1999. Discrete breathers in systems with homogeneous potentials: analytic solutions. Phys Rev Lett, 83, 248.

[12] RS MacKay \& S Aubry. 1994. Proof of existence of breathers for time-reversible or Hamiltonian networks of weakly coupled oscillators. Nonlinearity, 7, 1623.

[13] S Aubry \& G Abramovici. 1990. Chaotic trajectories in the standard map: the concept of antiintegrability. Physica D, 43, 199.

[14] S Flach \& CR Willis. 1993. Localized excitations in a discrete Klein-Gordon system. Phys Lett A, $181,232$.

[15] S Flach, CR Willis \& E Olbrich. 1994. Integrability and localized excitations in nonlinear discrete systems. Phys Rev E 49,836.

[16] S Flach, K Kladko \& C R Willis. 1994. Localized excitations in two-dimensional Hamiltonian lattices. Phys Rev E, 50, 2293.

[17] S Aubry, G Kopidakis \& V Kadelburg. 2001. Variational proof for hard discrete breathers in some classes of Hamiltonian dynamical systems. Disc Cont Dyn Sys B, 1, 271.

[18] G James. 2001. Existence of breathers on FPU lattices. C R Acad Sci Paris, 332, 581.

[19] KW Sandusky \& JB Page. 1994. Interrelation between the stability of extended normal modes and the existence of intrinsic localized modes in nonlinear lattices with realistic potentials. Phys Rev $B, \mathbf{5 0}, 866$.

[20] S Flach. 1996. Tangent bifurcation of band edge plane waves, dynamical symmetry breaking and vibrational localization. Physica D, 91, 223.

[21] S Flach, K Kladko \& RS Mackay. 1997. Energy thresholds for discrete breathers in one-, two-, and three-dimensional lattices. Phys Rev Lett, 78, 1207.

[22] M Kastner. 2004. Energy thresholds for discrete breathers. Phys Rev Lett, 92, 104301.

[23] FM Russell. 1988. Identification and selection criteria for charged lepton tracks in mica. Nucl Tracks Radiat Meas, 15, 41.

[24] JL Marin, JC Eilbeck \& FM Russell. 2000. 2D breathers and applications, Nonlinear Science at the Dawn of the 21st Century, Eds: PL Christiansen \& MP Soerensen, Springer, Berlin 293-306.

[25] JL Marin, JC Eilbeck \& FM Russell. 1998. Localised moving breathers in a 2D hexagonal lattice. Phys Lett A, 248, 225.

[26] FM Russell \& DR Collins. 1995. Lattice-solitons in radiation damage. Nucl Inst Meth Phys Res $B, \mathbf{1 0 5}, 30$.

[27] JL Marin, FM Russell \& JC Eilbeck. 2001. Breathers in cuprate-like lattices. Phys Lett A, 281, 21.

[28] M Remoissenet. 1986. Low-amplitude breather and envelope solitons in quasi-one-dimensional physical models. Phys Rev B, 33, 2386.

[29] Y Kivshar \& B Malomed. 1989. Dynamics of solitons in nearly integrable systems. Rev Mod Phys, 61, 763.

[30] IA Butt \& JAD Wattis. 2006. Moving breathers in the Fermi-Pasta-Ulam lattice. Submitted.

[31] RY Chiao, E Garmire \& CH Townes. 1964. Self-trapping of optical beams. Phys Rev Lett, 13, 479.

[32] C Sulem \& P-L Sulem. 1999. The Nonlinear Schrödinger Equation, Springer, New York. 
[33] WA Strauss. 1989. Nonlinear Wave Equations, American Mathematical Society, USA.

[34] M Remoissenet. 1999. Waves Called Solitons, Springer-Verlag, Berlin.

[35] G Fibich \& G Papanicolaou. 1999. Self-focusing in the perturbed and unperturbed nonlinear Schrödinger equation in critical dimension. SIAM J Appl Math, 60, 183.

[36] JJ Rasmussen \& K Rypdal. 1989. Blow-up in nonlinear Schrödinger equations I: a general review. Physica Scripta, 33, 481.

[37] YS Kivshar \& DE Pelinovsky. 2000. Self-focusing and transverse instabilities of solitary waves. Phys Rep, 331, 117.

[38] J Schjødt-Eriksen, YB Gaididei \& PL Christiansen. 2001. Collapse arresting in an inhomogeneous two-dimensional nonlinear Schrödinger model. Phys Rev E, 64, 066614.

[39] FK Abdullaev, BB Baizakov \& M Salerno. 2003. Stable two-dimensional dispersion-managed soliton. Phys Rev B, 68, 066605.

[40] VI Karpman. 1996. Stabilization of soliton instabilities by higher-order dispersion: fourth-order nonlinear Schrödinger-type equations. Phys Rev E, 53, R1336.

[41] TA Davydova, AI Yakimenko \& YA Zaliznyak. 2003. Two-dimensional solitons and vortices in normal and anomalous dispersive media. Phys Rev E, 67, 026402.

[42] EA Kuznetsov, AM Rubenchik \& VE Zakharov. 1986. Soliton stability in plasmas and hydrodynamics. Phys Rep, 142, 103.

[43] GH Derrick. 1964. Comments on nonlinear wave equations as models for elementary particles. $J$ Math Phys, 5, 1252.

[44] G Fibich \& G Papanicolaou. 1998. A modulation method for self-focusing in the perturbed critical nonlinear Schrdinger equation. Phys Lett A, 239, 167.

[45] HC Yuen \& BM Lake. 1975. Nonlinear deep water waves: theory and experiment. Physics of Fluids, 18, 956.

[46] V E Zakharov. 1972. Collapse of Langmuir waves. Zh Eksp Teor Fiz, 62, 1745, [Sov Phys JETP, 35, 908].

[47] JC Eilbeck. 1991. Numerical studies of solitons on lattices. In Nonlinear Coherent Structures in Physics and Biology, Lecture Notes in Physics Vol 393, Eds M Remoissenet \& M Peyrard, Springer-Verlag, Berlin.

[48] JAD Wattis. 1994. Solitary waves on a two-dimensional lattice. Physica Scripta, 50, 238.

[49] S Flach \& CR Willis. 1994. Movability of localized excitations in nonlinear discrete systems: a separatrix problem. Phys Rev Lett, 72, 1777.

[50] S Aubry \& T Cretegny. 1998. Mobility and reactivity of discrete breathers. Physica D, 119, 34.

[51] S Flach \& A Gorbach. 2005. Discrete breathers in Fermi-Pasta-Ulam lattices. Chaos, 15, 015112.

[52] JAD Wattis. 1996. Variational approximations to breathers in the discrete sine-Gordon equation II: moving breathers and Peierls-Nabarro energies. Nonlinearity, 9, 1583. 